$51^{\text {st }}$ AIAA Aerospace Sciences Meeting and Exhibit, 7-10 January 2013, Grapevine, Texas.

\title{
On the Resolution Necessary to Capture Dynamics of Unsteady Detonation
}

\author{
Christopher M. Romick* \\ University of Notre Dame, Notre Dame, Indiana, 46556-5637, USA \\ Tariq D. Aslam ${ }^{\dagger}$ \\ Los Alamos National Laboratory, Los Alamos, New Mexico 87545, USA \\ Joseph M. Powers ${ }^{\ddagger}$ \\ University of Notre Dame, Notre Dame, Indiana, 46556-5637, USA
}

\begin{abstract}
The dynamics of one-dimensional, overdriven, hydrogen-air detonations predicted in the inviscid limit as well as with the inclusion of mass, momentum, and energy diffusion were investigated. The effect of resolution was studied for both shock-capturing and shockfitting in the inviscid limit, and it was found that shock-capturing required four times the amount of resolution of shock-fitting to predict the essential dynamics. Harmonic analysis was used to examine how the long time dynamics changed as the overdrive was varied in both the inviscid limit and the viscous analog. As the overdrive is lowered, the pulsation's fundamental frequency shifts smoothly from $1.03 \mathrm{MHz}$ at an overdrive of $f=1.12$ to $0.71 \mathrm{MHz}$ at an overdrive of $f=1.035$ before making a sudden jump to $0.11 \mathrm{MHz}$ at an overdrive of $f=1.029$, where overdrive is $f=D_{o}^{2} / D_{C J}^{2}, D_{o}$ the initial detonation velocity, and $D_{C J}$ the Chapman-Jouguet velocity. It was found that viscous effects reduce the magnitude of the pulsations. As the strength of the inherent instability grows, due to interaction of the hydrodynamics and chemical reactions, the effect of viscosity is reduced.
\end{abstract}

\section{Introduction}

Detonations are an inherently multi-scaled reactive flow phenomenon, where the exothermic energy release due to chemical reactions contributes to driving a shock. These reactions can evolve on disparate scales; for a steadily traveling Chapman-Jouguet (CJ) hydrogen-air detonation propagating into an ambient state at atmospheric conditions, this range is from $10^{-5} \mathrm{~cm}$ to $10^{0} \mathrm{~cm}$ at equilibrium and can be even larger away from equilibrium. ${ }^{1}$ The scales are due to the averaged representation of molecular collision model in which the underlying length scale is the mean-free path. ${ }^{2}$ This wide range of scales needs to be resolved to accurately predict detonation dynamics. Therefore, much of the work in detonation modelling has been done with simplified kinetics in the inviscid limit. ${ }^{3}$

However, there have been some studies conducted using detailed kinetics, most of which have been restricted to one-dimension or low pressure due to the computational cost. One of the first performed was done by Sussman, ${ }^{4}$ who examined the dynamics of one-dimensional hydrogen-air detonations in the inviscid limit at ambient pressure of $0.421 \mathrm{~atm}$. In the inviscid limit, Eckett ${ }^{5}$ found while studying overdriven, hydrogen-oxygen detonations propagating into an ambient atmospheric pressure in one dimension that it was necessary to have a minimum of 150 points in the induction zone. Singh et al. ${ }^{6}$ simulated the development of a viscous detonation in a hydrogen-oxygen-argon mixture while using an operator-spliting technique and an intrinsic low-dimensional manifold. Yungster and Radhakrishan ${ }^{7}$ found, that while using shock-capturing in the inviscid limit, it was necessary to use a resolution on the order of a micron to capture the dynamics

*Ph.D. Candidate, Department of Aerospace and Mechanical Engineering, and AIAA Student Member. cromick@nd.edu

$\dagger$ Technical Staff Member, Weapons Experiments Division, and AIAA Member.

$\ddagger$ Professor, Department of Aerospace and Mechanical Engineering, and AIAA Associate Fellow. 
of a one-dimensional, hydrogen-air detonation. Examining various one-dimensional, inviscid, overdriven hydrogen-air detonations, Daimon and Matsuo ${ }^{8}$ predicted long time behavior of the detonation that becomes more complex as the overdrive is lowered, similar to that observed by Sussman, Eckett, and Yungster and Radhakrishan for other hydrogen-based mixtures. Several two-dimensional studies have been performed at low pressure for highly-dilute mixtures of hydrogen-oxygen-argon using detailed kinetics in the inviscid limit examining the development of detonation cell-size. ${ }^{9-11}$ Ziegler et al. ${ }^{12}$ examined a viscous, double Mach reflection detonation in a hydrogen-air mixture and found that even with a finest resolution near a micron that their results showed the resolution was insufficient. In the inviscid limit, Taylor et al. ${ }^{13}$ compared the intrinsic cell sizes of hydrogen-air detonation using detailed kinetics with one-step kinetics at ambient atmospheric conditions.

The majority of the work performed in modeling detonations has been done in the inviscid limit, as it is often suggested that viscous effects are negligible. However, there are indications that viscosity has a role. Furthermore, in detonations propagating into atmospheric ambient mixtures there are reaction dynamics and steep gradients near a micron scale suggesting that physical diffusion could play an important role. In fact, while using resolutions near a micron in a three-dimensional inviscid simulation to predict the behavior of spinning detonations, Tsuboi et al. ${ }^{14}$ report that while some structures appear to converge, "The present results cannot resolve such cross-hatchings in the ribbon because of a lack of grid resolution." Deiterding ${ }^{15}$ also reported that the interactions between chemistry and hydrodynamic flow in inviscid detonations "in general exhibit a strong dependency on the mesh spacing." In a two-dimensional study of detonation patterns, Powers ${ }^{16}$ found that patterns were strongly grid-dependent for the reactive Euler equations, but for the reactive Navier-Stokes equations relaxed to a grid-independent dissipative structure. This suggests that numerical diffusion is playing a significant role. While studying detonation propagation in micro-channels using a simplified kinetics model, Kivotides ${ }^{17}$ concluded that viscous effects can alter the transverse wave behavior. Using the one-step model, it was shown that when the viscous length scale is similar to that of the finest reaction scale, viscous effects play a critical role in determining the long time behavior of the detonation. ${ }^{18}$ In particular, viscous effects delay the transition to instability, and can play a dramatic role for detonations in the highly nonlinear regime characterized by oscillations at multiple frequencies. Mazaheri et al. ${ }^{19}$ demonstrated that diffusion can play an important role in regions of high-resolutions. Furthermore recent calculations done by Al-Khateeb et al. ${ }^{20}$ indicate that the fine reaction length scales present in hydrogen-air mixtures can have time scales associated with them at which both chemistry and diffusion become important. Therefore, the introduction of grid-independent physical diffusion should be considered.

The goal of this paper is to demonstrate how numerical diffusion from shock-capturing and the addition of physical mass, momentum, and energy diffusion affect the predicted behavior of one-dimensional hydrogenair detonations using a detailed kinetics mechanism. The plan of this paper is as follows. The mathematical model is presented followed by a description of the computational methods. This model is used to predict the dynamics of several overdriven hydrogen-air detonations. It will be shown that as the initial overdrive is decreased, the long time behavior becomes more complex, in agreement with similar previous studies performed in the inviscid limit. Additionally, the long time behavior will be examined using harmonic analysis to give further insight into how the behavior evolves with respect to the initial overdrive. Furthermore, this type analysis is used to reveal how the energy is distributed in the frequency domain, and reveals the differences between inviscid, shock-fitted calculations and the shock-capturing and viscous analogs. As seen in the one-step model, the addition of viscous effects will be shown to have a stabilizing effect in the weakly unstable regime. However, as the overdrive is lowered, stabilization due to the addition of viscosity is weakened due to the strengthening intrinsic instability.

\section{Model}

\section{A. Mathematical model}

The governing equations are the reactive, compressible Navier-Stokes equations and are expressed as

$$
\begin{aligned}
\frac{\partial \rho}{\partial t}+\nabla \cdot(\rho \mathbf{u}) & =0, \\
\frac{\partial}{\partial t}(\rho \mathbf{u})+\nabla \cdot(\rho \mathbf{u u}+p \mathbf{I}-\boldsymbol{\tau}) & =\mathbf{0},
\end{aligned}
$$




$$
\begin{aligned}
\frac{\partial}{\partial t}\left(\rho\left(e+\frac{\mathbf{u} \cdot \mathbf{u}}{2}\right)\right)+\nabla \cdot\left(\rho \mathbf{u}\left(e+\frac{\mathbf{u} \cdot \mathbf{u}}{2}\right)+(p \mathbf{I}-\boldsymbol{\tau}) \cdot \mathbf{u}+\mathbf{q}\right) & =0 \\
\frac{\partial}{\partial t}\left(\rho Y_{i}\right)+\nabla \cdot\left(\rho \mathbf{u} Y_{i}+\mathbf{j}_{i}\right) & =\bar{M}_{i} \dot{\omega}_{i}
\end{aligned}
$$

where Eqs. (1)-(4) represent the conservation of mass, linear momenta, energy and the evolution of species. The independent variables are $t$, the temporal coordinate, and $\mathbf{x}$ the spatial coordinates. The dependent variables are the mixture mass density, $\rho$, the mixture velocity components, $\mathbf{u}$, the mixture pressure, $p$, the viscous stress tensor, $\boldsymbol{\tau}$, the specific internal energy of the mixture, $e$, the total heat flux vector, q, the mass fraction, $Y_{i}$, the diffusive mass flux, $\mathbf{j}_{i}$, the molecular mass, $\overline{M_{i}}$, and the molar production rate per unit volume, $\dot{\omega}_{i}$, for the $i^{\text {th }}$ specie. The $\nabla$ symbol is the gradient operator, and $\mathbf{I}$ is the identity matrix. Equations (11) and (3) are scalar equations, and Eqs. (2) and (4) vector equations of lengths $d$ and $N-1$, respectively, where $\mathrm{d}$ is the dimension of the problem, and $N$ is the number of species. To close the system, constitutive relations must be specified; the following constitutive relations have been chosen for a mixture of $N$ species composed of $L$ elements interacting in $J$ reactions:

$$
\begin{aligned}
& \mathbf{j}_{i}=\rho \sum_{\substack{k=1 \\
k \neq i}}^{N} \frac{\overline{M_{i}} D_{i k} Y_{k}}{\bar{M}}\left(\frac{\nabla y_{k}}{y_{k}}+\left(1-\frac{\overline{M_{k}}}{\bar{M}}\right) \frac{\nabla p}{p}\right)-\frac{D_{i}^{T} \nabla T}{T}, \\
& \boldsymbol{\tau}=\mu\left(\nabla \mathbf{u}+(\nabla \mathbf{u})^{T}-\frac{2}{3}(\nabla \cdot \mathbf{u}) \mathbf{I}\right) \\
& \mathbf{q}=-\mathrm{k} \nabla T+\sum_{i=1}^{N} \mathbf{j}_{i} h_{i}-\mathcal{R} T \sum_{i=1}^{N} \frac{D_{i}^{T}}{\overline{M_{i}}}\left(\frac{\nabla y_{i}}{y_{i}}+\left(1-\overline{\overline{M_{i}}}\right) \frac{\nabla p}{\bar{M}}\right), \\
& y_{i}=\frac{\bar{M}}{\overline{M_{i}}} Y_{i} \\
& \bar{M}=\left(\sum_{i=1}^{N} \frac{Y_{i}}{\overline{M_{i}}}\right)^{-1} \\
& p=\rho R T \text {, } \\
& R=\frac{\mathcal{R}}{\bar{M}}, \\
& h_{i}=h_{i}^{r e f}+\int_{T_{r e f}}^{T} c_{P, i}(\hat{T}) d \hat{T} \\
& e=\sum_{i=1}^{N} Y_{i}\left(h_{i}-\frac{\mathcal{R} T}{\bar{M}_{i}}\right) \\
& \dot{\omega}_{i}=\sum_{j=1}^{J} \nu_{i j} r_{j} \\
& r_{j}=k_{j}\left(\prod_{i=1}^{N}\left(\rho \frac{Y_{i}}{\overline{M_{i}}}\right)^{\nu_{i j}^{\prime}}-\frac{1}{K_{j}^{c}} \prod_{i=1}^{N}\left(\rho \frac{Y_{i}}{\overline{M_{i}}}\right)^{\nu_{i j}^{\prime \prime}}\right), \\
& k_{j}=a_{j} T^{\beta_{j}} \exp \left(\frac{-\bar{E}_{j}}{\mathcal{R} T}\right), \\
& K_{j}^{c}=\left(\frac{p^{r e f}}{\mathcal{R} T}\right)^{\sum_{i=1}^{N} \nu_{i j}} \exp \left(\frac{-\sum_{i=1}^{N} \bar{g}_{i}^{o} \nu_{i j}}{\mathcal{R} T}\right), \\
& \sum_{i=1}^{N} Y_{i}=1 \\
& \sum_{i=1}^{N} \mathbf{j}_{i}=\mathbf{0} \\
& \sum_{i=1}^{N} \phi_{l i} \nu_{i j}=0 \\
& \nu_{i j}=\nu_{i j}^{\prime \prime}-\nu_{i j}^{\prime},
\end{aligned}
$$


where $\bar{M}$ is the mixture molecular mass, $D_{i k}$ the multi-component diffusion coefficient between the $i^{\text {th }}$ and $k^{t h}$ species, $y_{i}$ the mole fraction of the $i^{t h}$ specie, $D_{i}^{T}$ the thermal diffusion coefficient of the $i^{t h}$ specie, $T$ the temperature, $\mu$ the dynamic viscosity of the mixture, $k$ the thermal conductivity of the mixture, $R$ the mixture gas constant, $c_{P, i}$ the specific heat at constant pressure, $h_{i}$ the specific enthalpy, and $h_{i}^{r e f}$ the specific enthalpy evaluated at the reference pressure of the $i^{\text {th }}$ specie, $\mathcal{R}$ the universal gas constant which is $8.314 \times 10^{7} \mathrm{erg} /($ mole $K), p^{r e f}$ the reference pressure which is $1.01325 \times 10^{6}$ dyne $/ \mathrm{cm}^{2}, T^{r e f}$ the reference temperature which is $298.15 K, \nu_{i j}$ the net stoichiometric coefficient, $\nu_{i j}^{\prime}$ the forward stoichiometric coefficient, and $\nu_{i j}^{\prime \prime}$ the reverse stoichiometric coefficient of the $i^{t h}$ specie in the $j^{\text {th }}$ reaction, $r_{j}$ the reaction rate, $k_{j}$ the Arrhenius model for the reactions' temperature sensitivity, $a_{j}$ the collision frequency factor, $\beta_{j}$ the temperature exponent, $\bar{E}_{j}$ the activation energy, and $K_{j}^{c}$ the equilibrium constant for the $j^{t h}$ reaction, $\bar{g}_{i}^{o}$ the $i^{t h}$ species' chemical potential at the reference pressure, and $\phi_{l i}$ the number of atoms of element $l$ in the $i^{\text {th }}$ species. Equations (5)-(7) give constitutive relations for mass, momentum, and energy diffusion, which are an extended Fick's law, a Newtonian stress-strain rate relation, and an extended Fourier's law. The form of both Fourier's and Fick's law is appropriate for a mixture of ideal gases, as detailed in a derivation by Merk ${ }^{21}$ and summarized by Kee et al..$^{22}$ and accounts for multicomponent mass diffusion as well as Soret and DuFour effects. Equation (6) has used Stokes' assumption. The reaction and mixture properties are evaluated using the CHEMKIN ${ }^{23}$ and TRANSPORT ${ }^{22}$ packages, respectively. Note for inviscid calculations, all diffusion coefficients, viscosity, and thermal conductivity are taken to be zero.

The use of continuum models like the Euler or Navier-Stokes equations is sometimes called into question at the fine scales needed in detonation modeling. The mean-free path is the cut-off minimum length scale associated with continuum theories. Using the simple definition given by Vincenti and Kruger ${ }^{24}$ the mean-free path in a typical overdriven detonation is $\lambda=\bar{M} /\left(\sqrt{2} \pi \mathcal{N}_{A} \rho d^{2}\right) \approx 3 \times 10^{-6} \mathrm{~cm}$, where $\bar{M} \approx 20.911 \mathrm{~g} /$ mole, $\mathcal{N}_{A}=6.022 \times 10^{23}$ molecules $/$ mole is Avogadro's constant, $\rho \approx 2.00 \times 10^{-3} \mathrm{~g} / \mathrm{cm}^{3}$, and $d \approx 3.621 \times 10^{-8} \mathrm{~cm}$ is the molecular collision diameter. Using an approximate kinematic viscosity in the burned gases of $\nu=7 \times 10^{-1} \mathrm{~cm}^{2} / \mathrm{s}$ and a frozen sound speed in the reacted zone of $c \approx 9 \times 10^{4} \mathrm{~cm} / \mathrm{s}$, an approximate viscous length scale is $\nu / c=7.8 \times 10^{-6} \mathrm{~cm} \approx \mathcal{O}\left(10^{-5} \mathrm{~cm}\right)$. The finest reaction length scale was calculated by the spatial eigenvalue analysis method of Powers and Paolucci and was found to be near $10^{-4} \mathrm{~cm}$. The finest reaction length scales are near those of the viscous scales, and the viscous terms in the Navier-Stokes equations may become important. The Knudsen number, $K n=\lambda / L$, where $L$ is a representative length scale of the problem, is an indicator of how well continuum model captures the physics. Application of continuum models requires $K n \lesssim \mathcal{O}(1)$. The respective Knudsen numbers for the reactive and viscous length scales yield $K n=3 \times 10^{-2}$ and $K n=4 \times 10^{-1}$, which are both smaller than $\mathcal{O}(1)$. Therefore, the inherent physics in the inviscid and viscous models are within the continuum regime.

\section{B. Computational method}

Both inviscid and viscous calculations were performed. For the inviscid calculations two methods were explored. The first was a shock-fitting strategy, adapted from Henrick et al. ${ }^{25}$ to allow for an arbitrary number of chemical species as well as equations of state for ideal mixtures of calorically imperfect gases, which enforces the shocked-state at the boundary. The underlying numerical scheme is a nominally fifthorder spatial scheme which utilizes a central spatial scheme with special one-sided differences near the shock, as the Euler equations were transformed into the shock-attached frame. The more common practice of shockcapturing was used as a second method of investigation in the inviscid limit and made use of a nominally second-order min-mod spatial scheme. Both strategies were applied using a uniform grid, using a local LaxFriedrichs scheme. The viscous calculations were performed using the Wavelet Adaptive Multiresolution Representation (WAMR) method, first developed by Vasilyev and Paolucci. ${ }^{26,27}$ This is an adaptive mesh refinement technique, based on wavelet functions. These functions have compact support in both space and scale, which allows for a large compression of data. Thus, many less points are needed to accurately represent a flow-field relative to a wide variety of other approaches. It utilizes a user-specified control parameter that correlates to the error tolerated in the solution allowing unnecessary points to be discarded. The WAMR method has been applied successfully to a number of fluids problems. ${ }^{6,28-33}$ For a detailed description of the method in its current form, see Paolucci et al.. ${ }^{33-35}$ In both the inviscid and viscous cases, temporal integration was accomplished using a nominally fifth-order Runge-Kutta scheme. 
Table 1. Hydrogen-air reaction mechanism

\begin{tabular}{|c|c|c|c|c|}
\hline $\mathrm{j}$ & Reaction & $a_{j}\left(\left(\text { mole } / \mathrm{cm}^{3}\right)^{\left(1-\sum_{i=1}^{N} \nu_{i j}^{\prime}\right)} /\left(K^{\beta_{j}} s\right)\right)$ & $\beta_{j}$ & $E_{j}\left(\frac{c a l}{m o l e}\right)$ \\
\hline 1 & $\mathrm{H}_{2}+\mathrm{O}_{2} \rightleftharpoons 2 \mathrm{OH}$ & $1.70 \times 10^{13}$ & 0.00 & 47780 \\
\hline 2 & $\mathrm{OH}+\mathrm{H}_{2} \rightleftharpoons \mathrm{H}_{2} \mathrm{O}+\mathrm{H}$ & $1.17 \times 10^{9}$ & 1.30 & 3626 \\
\hline 3 & $\mathrm{H}+\mathrm{O}_{2} \rightleftharpoons \mathrm{OH}+\mathrm{O}$ & $5.13 \times 10^{16}$ & -0.816 & 16507 \\
\hline 4 & $O+H_{2} \rightleftharpoons O H+H$ & $1.80 \times 10^{10}$ & 1.00 & 8826 \\
\hline 5 & $H+\mathrm{O}_{2}+M \rightleftharpoons \mathrm{HO}_{2}+M^{a}$ & $2.10 \times 10^{18}$ & -1.00 & 0 \\
\hline 6 & $\mathrm{H}+\mathrm{O}_{2}+\mathrm{O}_{2} \rightleftharpoons \mathrm{HO}_{2}+\mathrm{O}_{2}$ & $6.70 \times 10^{19}$ & -1.42 & 0 \\
\hline 7 & $\mathrm{H}+\mathrm{O}_{2}+\mathrm{N}_{2} \rightleftharpoons \mathrm{HO}_{2}+\mathrm{N}_{2}$ & $6.70 \times 10^{19}$ & -1.42 & 0 \\
\hline 8 & $\mathrm{OH}+\mathrm{HO}_{2} \rightleftharpoons \mathrm{H}_{2} \mathrm{O}+\mathrm{O}_{2}$ & $5.00 \times 10^{13}$ & 0.00 & 1000 \\
\hline 9 & $\mathrm{H}+\mathrm{HO}_{2} \rightleftharpoons 2 \mathrm{OH}$ & $2.50 \times 10^{14}$ & 0.00 & 1900 \\
\hline 10 & $\mathrm{O}+\mathrm{HO}_{2} \rightleftharpoons \mathrm{O}_{2}+\mathrm{OH}$ & $4.80 \times 10^{13}$ & 0.00 & 100 \\
\hline 11 & $2 \mathrm{OH} \rightleftharpoons \mathrm{O}+\mathrm{H}_{2} \mathrm{O}$ & $6.00 \times 10^{8}$ & 1.30 & 0 \\
\hline 12 & $H_{2}+M \rightleftharpoons H+H+M^{b}$ & $2.23 \times 10^{12}$ & 0.50 & 92600 \\
\hline 13 & $\mathrm{O}_{2}+M \rightleftharpoons O+O+M$ & $1.85 \times 10^{11}$ & 0.50 & 95560 \\
\hline 14 & $H+\mathrm{OH}+\mathrm{M \rightleftharpoons} \mathrm{H}_{2} \mathrm{O}+M^{c}$ & $7.50 \times 10^{23}$ & -2.60 & 0 \\
\hline 15 & $\mathrm{H}+\mathrm{HO}_{2} \rightleftharpoons \mathrm{H}_{2}+\mathrm{O}_{2}$ & $2.50 \times 10^{13}$ & 0.00 & 700 \\
\hline 16 & $\mathrm{HO}_{2}+\mathrm{HO}_{2} \rightleftharpoons \mathrm{H}_{2} \mathrm{O}_{2}+\mathrm{O}_{2}$ & $2.00 \times 10^{12}$ & 0.00 & 0 \\
\hline 17 & $\mathrm{H}_{2} \mathrm{O}_{2}+\mathrm{M} \rightleftharpoons \mathrm{OH}+\mathrm{OH}+\mathrm{M}$ & $1.30 \times 10^{17}$ & 0.00 & 45500 \\
\hline 18 & $\mathrm{H}_{2} \mathrm{O}_{2}+\mathrm{H} \rightleftharpoons \mathrm{HO}_{2}+\mathrm{H}_{2}$ & $1.60 \times 10^{12}$ & 0.00 & 3800 \\
\hline 19 & $\mathrm{H}_{2} \mathrm{O}_{2}+\mathrm{OH} \rightleftharpoons \mathrm{H}_{2} \mathrm{O}+\mathrm{HO}_{2}$ & $1.00 \times 10^{13}$ & 0.00 & 1800 \\
\hline \multicolumn{5}{|c|}{ Enhanced third-body efficiencies with $M$ : } \\
\hline & \multirow{3}{*}{\multicolumn{4}{|c|}{$\begin{array}{l}M^{a}: \alpha_{H_{2} O}=21.0, \alpha_{H_{2}}=3.30, \alpha_{N_{2}}=0.00, \alpha_{O_{2}}=0.00 \\
M^{b}: \alpha_{H_{2} O}=6.00, \alpha_{H}=2.00, \alpha_{H_{2}}=3.00 \\
M^{c}: \alpha_{H_{2} O}=20.0\end{array}$}} \\
\hline & & & & \\
\hline & & & & \\
\hline
\end{tabular}
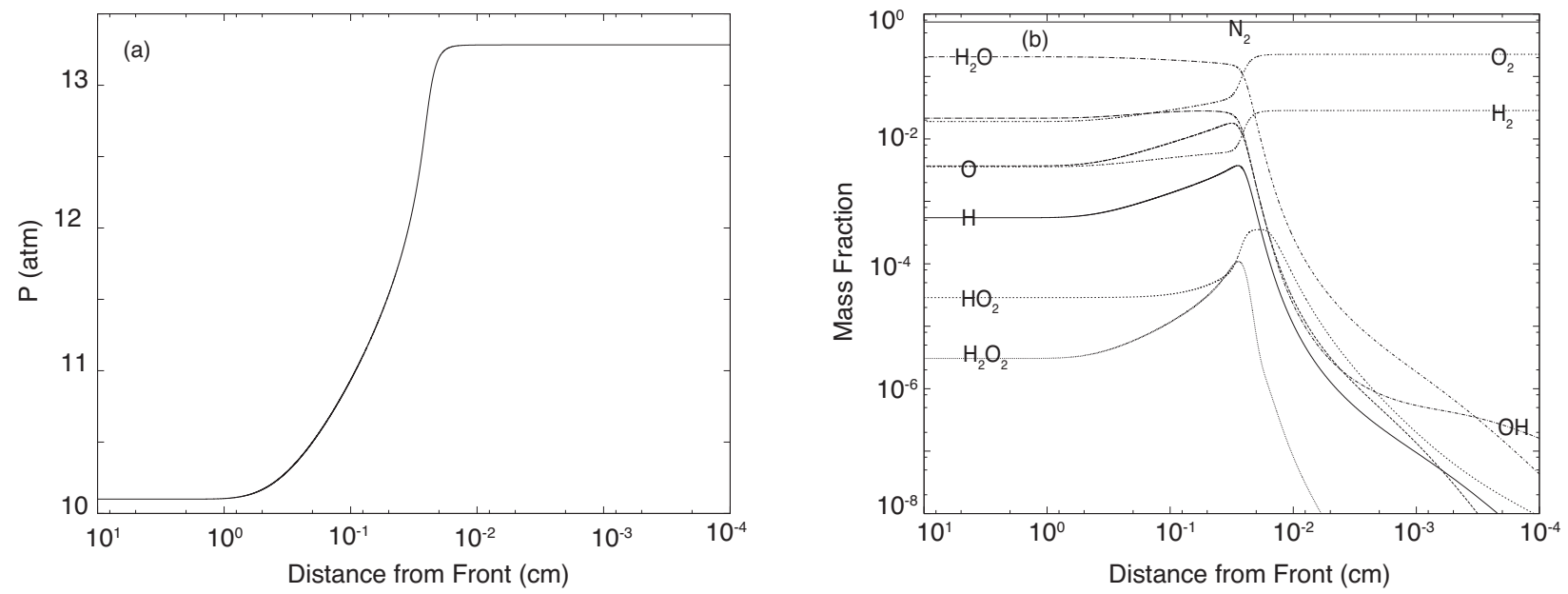

Figure 1: Structure of a steady inviscid, overdriven, hydrogen-air detonation, $f=1.15$ for (a) pressure and (b) mass fraction. The detonation front is traveling to the right.

\section{Physical Problem}

We consider a series of one-dimensional, overdriven, detonations of an initially stoichiometric mixture of hydrogen-air $\left(2 \mathrm{H}_{2}+\mathrm{O}_{2}+3.76 \mathrm{~N}_{2}\right)$ at ambient conditions of $293.15 \mathrm{~K}$ and $0.421 \mathrm{~atm}$. This choice of ambient conditions was made to enable comparison with previous calculations ${ }^{7,8}$ as well as the shock-induced combustion experiments of Lehr. ${ }^{36}$ The detailed kinetic mechanism employed in this study, shown in Table 1 was used by Powers and Paolucci ${ }^{1}$ and drawn originally from Miller et al.. ${ }^{37}$ It is composed of 19 reversible 
reactions, contains 9 species and 3 elements, where nitrogen is treated as a non-reacting species. At this ambient condition, this mechanism yields a $C J$ detonation velocity of $D_{C J} \sim 1972 \mathrm{~m} / \mathrm{s}$ which is similar to that found by Sussman, $D_{C J} \sim 1958 \mathrm{~m} / \mathrm{s}$.

Overdrives ranging from $1.018 \leq f \leq 1.15$ were examined, where the overdrive is $f=D_{o}^{2} / D_{C J}^{2}$ and $D_{o}$ the initial detonation velocity. For the viscous calculations presented the inviscid steady-state profile was used with a superimposed smooth transition from the shocked-state to the ambient condition over $5 \times 10^{-4} \mathrm{~cm}$. Using the WAMR method, all viscous detonations were performed using the user-specified error control parameter of $\epsilon=5 \times 10^{-4}$. This selection lead to a time-step on the order of $2 \times 10^{-12} s$ and the finest spatial resolution utilized of $6 \times 10^{-6} \mathrm{~cm}$, though finer scales were still allowed. All inviscid simulations were initialized using a resolved steady-state solution. As these calculations were performed on a uniform grid, the effect of resolution was examined. Typical simulation times for the inviscid calculations were $\sim 2 \mu s /$ per $24 C P U-h r s$. for $\Delta x=4 \mu m$ and for viscous calculations $\sim 1 \mu s /$ per $24 C P U-h r s$. on an AMD $2.4 G H z$ processor with a $512 k B$ cache.

\section{Results}

The typical steady-state structure of an overdriven hydrogen-air detonation is shown in Fig. 1 . This structure is calculated by integrating only spatial ordinary differential equations using a fifth-order RungeKutta scheme with a spatial discretization of $10^{-8} \mathrm{~cm}$. The pressure profile behind the front of a $f=1.15$, overdriven hydrogen-air detonation, shown in Fig. 1(a), begins to decrease near $x=10^{-2} \mathrm{~cm}$, indicating the end of the induction zone. The evolution of species behind the front, shown in Fig. 1(b), clearly depicts the disparity in reaction length scales. The final relaxation time occurs near $x=10^{0} \mathrm{~cm}$, whereas the first minor species, $O H$, begins to depart from power law growth near $x=5 \times 10^{-4} \mathrm{~cm}$. Thus in the inviscid limit, to accurately capture the dynamics of the detonation the spatial discretization needed is on the order of microns, and the macroscale must be on the order of centimeters or longer.

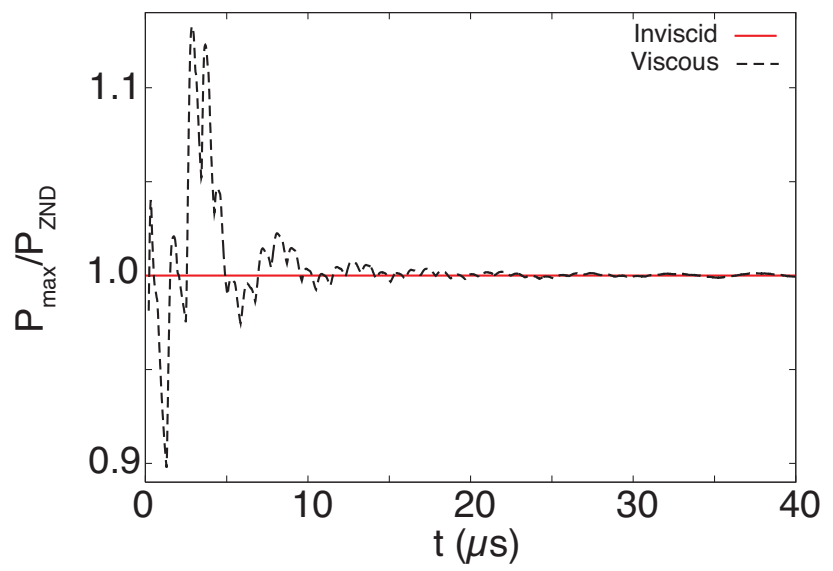

Figure 2: Time-dependent behavior for an overdriven, $f=1.15$, hydrogen-air detonation with an ambient state of $0.421 \mathrm{~atm}$ and $293.15 \mathrm{~K}$ for both the inviscid (shock-fitting, $\Delta x=4 \mu \mathrm{m}$ ) and viscous cases.

\section{A. Strongly overdriven detonations}

For sufficiently large overdrives, the detonation is stable at long times. There are large early perturbations present in the viscous case due to using an approximate viscous solution for initialization, as shown in Fig. 2 However, by about $40 \mu s$, the initial perturbations have decayed significantly, yielding a steadily propagating wave similar to that in the inviscid case for an overdrive of $f=1.15$.

\section{B. High-frequency oscillating detonations}

Below a critical overdrive, the detonation becomes unstable and begins to oscillate. A typical development of a high-frequency oscillating detonation in the inviscid limit, predicted using the shock-fitting and a spatial 


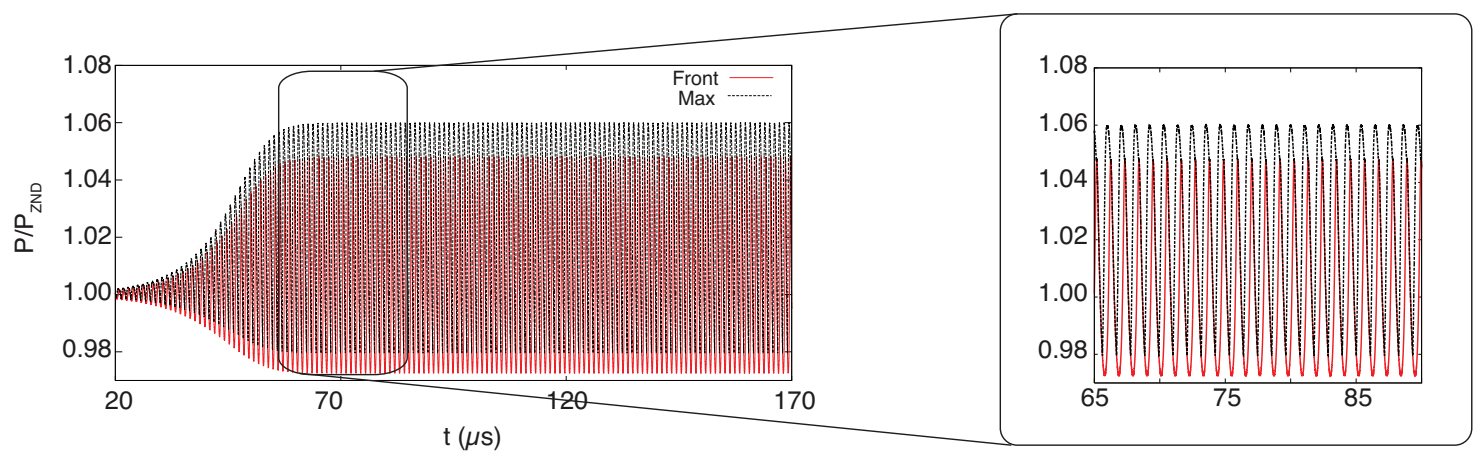

Figure 3: Time-dependent behavior for an overdriven, $f=1.10$, hydrogen-air detonation with an ambient state of $0.421 \mathrm{~atm}$ and $293.15 \mathrm{~K}$ in the inviscid limit using shock-fitting and a resolution of $\Delta x=4 \mu \mathrm{m}$.
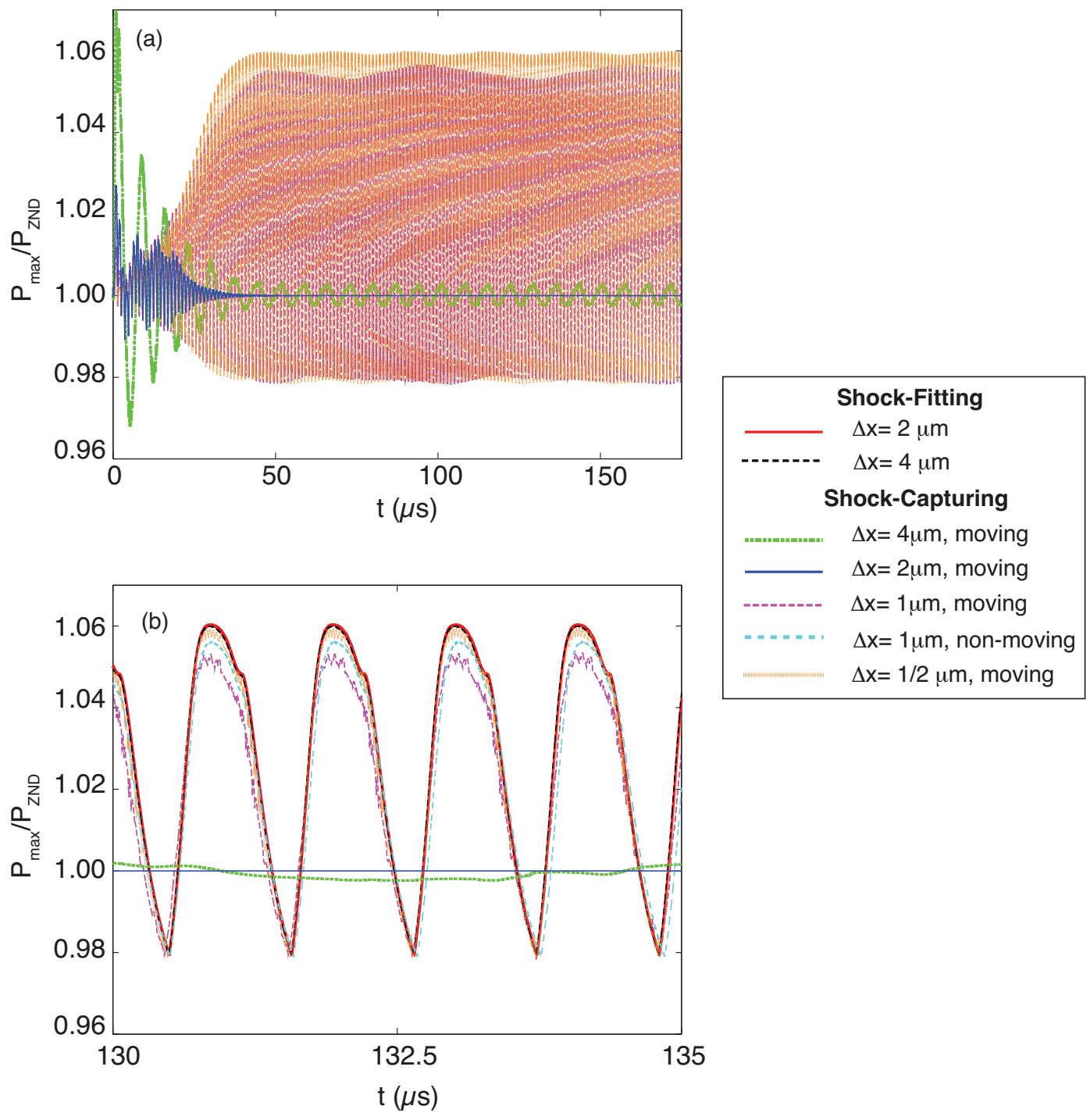

Figure 4: Time-dependent behavior for an overdriven, $f=1.10$, hydrogen-air detonation with an ambient state of $0.421 \mathrm{~atm}$ and $293.15 \mathrm{~K}$ in the inviscid limit using (a) shock-capturing and (b) its comparison over a shorter time period to shock-fitting predictions. 


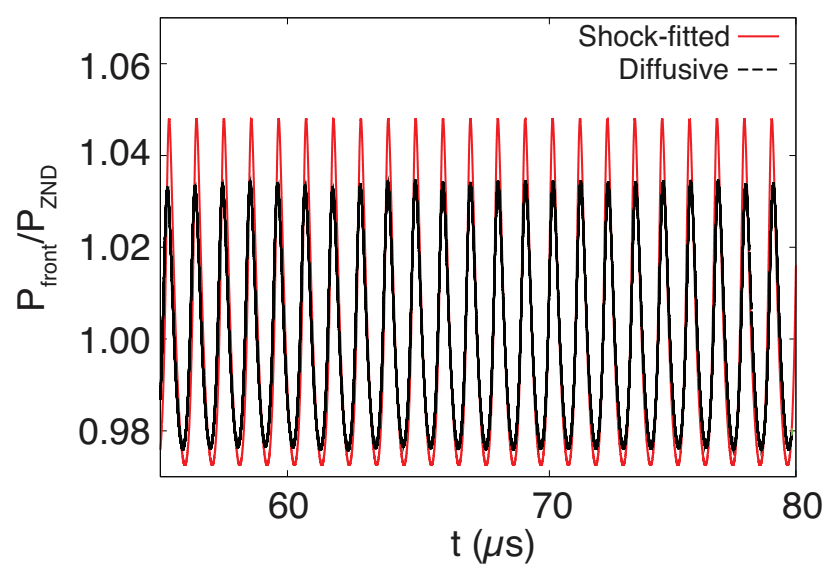

Figure 5: Time-dependent behavior for an overdriven, $f=1.10$, hydrogen-air detonation with an ambient state of $0.421 \mathrm{~atm}$ and $293.15 \mathrm{~K}$, for both the viscous and inviscid (shock-fitted, $\Delta x=4 \mu \mathrm{m}$ ) cases.

resolution of $\Delta x=4 \mu \mathrm{m}$, is shown in Fig. 3. At this overdrive, $f=1.10$, the detonation takes around $60 \mu s$ to saturate and becomes periodic with a frequency near $0.97 \mathrm{MHz}$. Using the more common technique of shock-capturing in a moving reference frame and utilizing the same spatial resolution used in the shockfitting case $(\Delta x=4 \mu m)$, the predicted behavior is qualitatively different. The predicted behavior misses the essential dynamics even though pulsations exist at long times, the amplitude and frequency are not in agreement with grid refinement or shock-fitting predictions. In fact, as shown in Fig. 4(a), at $\Delta x=2 \mu m$ these spurious predicted oscillations are completely damped by artificial diffusion. Moreover, only after refining the resolution to $\Delta x=1 \mu \mathrm{m}$, do the essential dynamics of the high-frequency oscillations begin to be captured properly. Even at this finer resolution, the use of shock-capturing in a moving reference frame predicts both a spurious lower frequency modulation of the detonation pressure (shown in Fig. 4(a)) and as well as a spurious higher frequency, as seen in Fig. 4(b). These spurious oscillations are most likely due to the slight mis-match of the detonation speed and frame of reference speed, a phenomenon first suggested by Quirk. ${ }^{38}$ Making use of shock-capturing in a non-moving reference frame removes both of these spurious oscillations, yet at a grid resolution of $\Delta x=1 \mu \mathrm{m}$, there remains a slight altering of the predicted frequency. This altering effect will be discussed further in section 2. It should be noted the profiles shown in Fig. 4)(b), of the various methods and resolutions, were shifted minutely in time for ease of comparison between the predictions. Examining the viscous analog of this particular overdrive, it is seen that it is also unstable. However as seen in Fig. 5. there is a significant damping of the amplitude of oscillations, nearly $20 \%$ for this particular overdrive. Nevertheless, the frequency of oscillations remains largely unaltered.

In his shock-induced combustion experiments around spherical projectiles in hydrogen-air mixtures, Lehr ${ }^{36}$ observed longitudinal oscillations at frequency of $1.04 \mathrm{MHz}$ for an inflow condition corresponding to an overdrive of $f \approx 1.10$, which is in agreement with the $0.97 \mathrm{MHz}$ predicted frequency in the current study. Yungster and Radhakrishan found a frequency of $1.06 \mathrm{MHz}$, for a similar overdrive of $f=1.09$ with an ambient temperature of $298 \mathrm{~K}$. Thus, it seems that the instability observed by Lehr in multiple dimensions is dominated by a one-dimensional instability mode and can be captured well by a one-dimensional model.

\section{Appearance of a low-frequency mode}

As the overdrive is lowered, the detonation becomes more unstable giving rise to amplitude growth of the pulsations. Lowering the overdrive yet further, detonations with two main frequencies begin to appear. These frequencies are about an order of magnitude different; with the higher being near $1 \mathrm{MHz}$ and the lower near $0.1 \mathrm{MHz}$. At a critical overdrive, near $f \approx 1.035$, in the inviscid limit the lower frequency begins to dominate at long times, and the pulsations grow in amplitude, as shown in Fig. 6 using shock-fitting with a resolution of $\Delta x=4 \mu \mathrm{m}$. Near this critical point, the competition between the high frequency and low frequency mode persists at long time. During the transition from the high frequency to low frequency, the pulsation amplitude grows as the inherent instability grows in strength and the detonation becomes more unstable. The viscous analog's behavior is compared with the prediction of shock-fitting in Fig. 7 where the 
inviscid case has been shifted in time by $270 \mu \mathrm{s}$. In this transition region, the viscous effects cause a decay of the lower frequency at longer times whereas in the inviscid case the pulsations continue to grow in time. The amount of energy that each of the frequencies carries will be discussed further in section 2. Longer times need to be further investigated to definitively say that the addition of physical diffusion removes the low frequency mode. A similar question that can be asked in the inviscid limit is whether or not the detonation continues to evolve towards a detonation exhibiting only the low frequency mode. This likewise can be answered by studying even longer times.

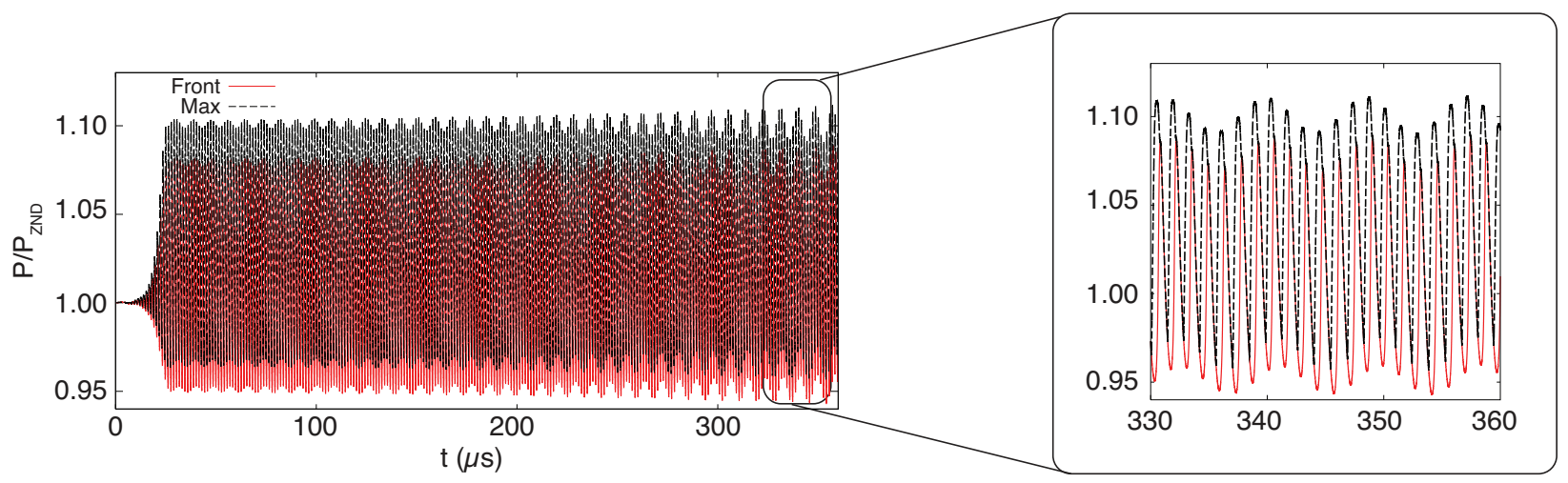

Figure 6: Time-dependent behavior for an overdriven, $f=1.035$, hydrogen-air detonation with an ambient state of $0.421 \mathrm{~atm}$ and $293.15 \mathrm{~K}$ in the inviscid limit using shock-fitting and a resolution of $\Delta x=4 \mu \mathrm{m}$.

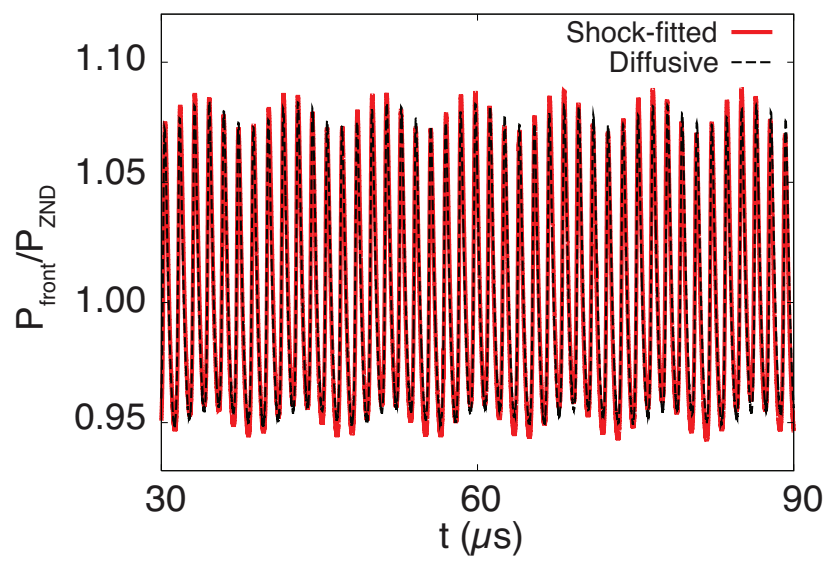

Figure 7: Time-dependent behavior for an overdriven, $f=1.035$, hydrogen-air detonation with an ambient state of $0.421 \mathrm{~atm}$ and $293.15 \mathrm{~K}$, for both the viscous and inviscid (shock-fitted, $\Delta x=4 \mu \mathrm{m}$ ) cases. The inviscid case has been shifted by $270 \mu \mathrm{s}$.

At a slightly lower overdrive of $f=1.023$, the frequency competition is shorter lived in time, and the low frequency mode dominates earlier. The prediction of the behavior at this overdrive for both shock-fitting and shock-capturing for various resolutions are shown in Fig. 8 (a). At long times, the low frequency-large pulsations dominate as seen in the saturation of the maximum pressure; yet, the higher frequency persists even at long times. This is seen in both the shock-fitting case shown and at the finer grids used in shockcapturing. As in the strictly high-frequency mode, a finer resolution is needed when using a shock-capturing scheme in a moving reference frame when compared with the predictions of shock-fitting. Using of the same resolution $(\Delta x=4 \mu \mathrm{m})$ for shock-capturing as shock-fitting, there is a dramatic over-prediction of the pulsation amplitude that persists through the duration of the simulation. Refining the resolution, to $\Delta x=2 \mu m$, begins to capture the competition of between the low and high-frequencies at early times; however, at later times an over-prediction of the detonation pressure is seen. In fact as was the case for the strictly high frequency mode, a more quantitative agreement with the shock-fitted results is not seen until the resolution is refined to $\Delta x=1 \mu \mathrm{m}$. At the finer resolutions, the behavior predicted by shock-capturing 

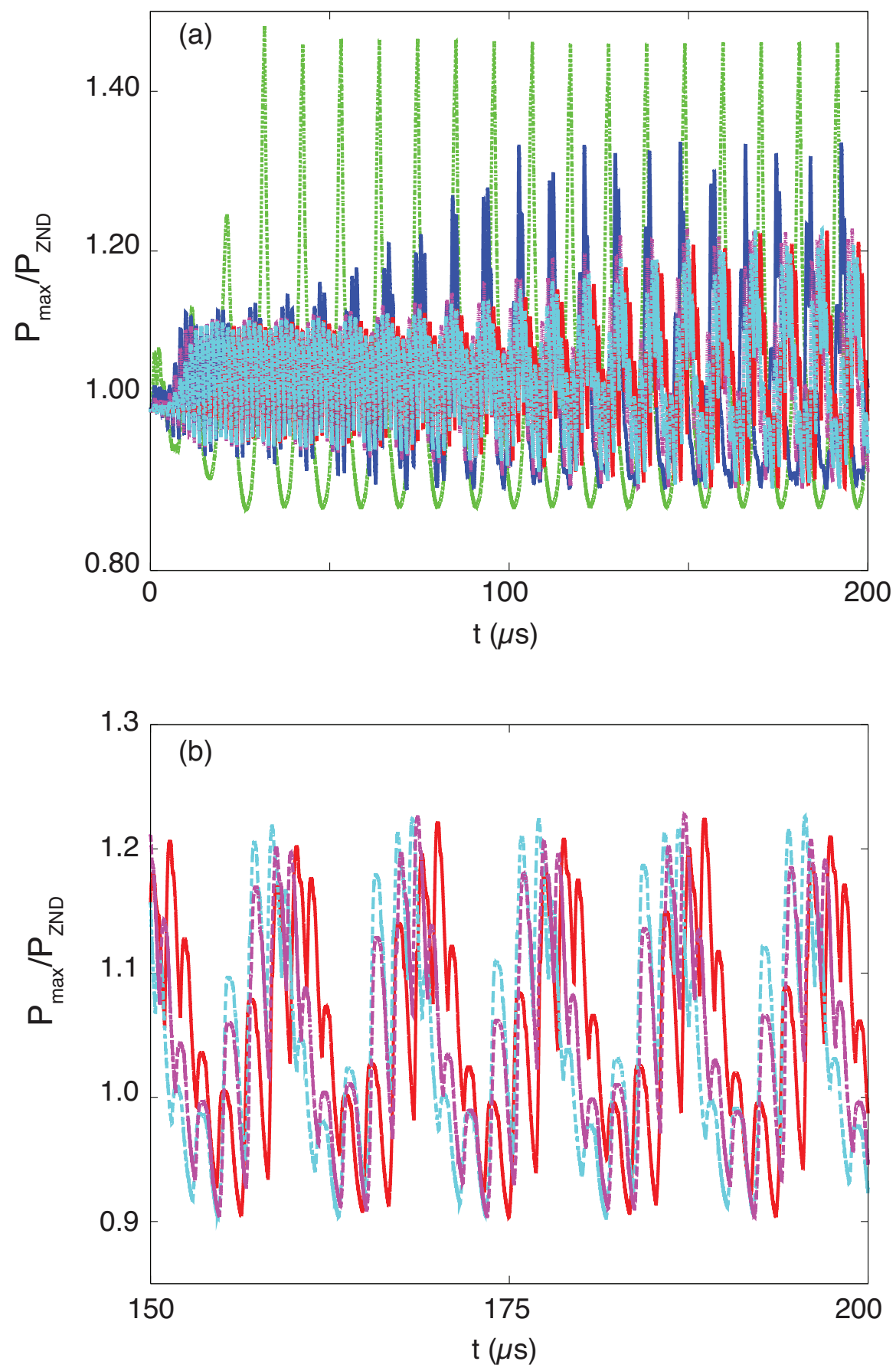

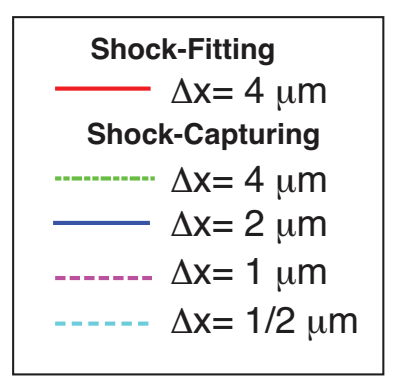

Figure 8: Time-dependent behavior for an overdriven, $f=1.023$, hydrogen-air detonation with an ambient state of $0.421 \mathrm{~atm}$ and $293.15 \mathrm{~K}$ in the inviscid limit using both shock-capturing and shock-fitting over (a) long time periods and (b) a zoomed comparison at long times. 
begins to converge with that predicted by shock-fitting as seen in the zoomed profile of detonation pressure versus time curve in Fig. 8 (b). The over-prediction of the detonation behavior is counter-intuitive as one would expect the larger numerical viscosity at coarser grids to add stability. However, since the grid is coarser the strength of the instability needed to facilitate the global instability is less as well. As the overdrive is lowered the strength of the intrinsic instability grows stronger, due to the interaction of the hydrodynamics and chemical reactions, and thus at the coarser resolution an easier opportunity for over-prediction of the pulsation amplitudes exists.

\section{Harmonic Analysis}

It can be difficult to understand how the energy of the pulsation cycle is distributed from pressure versus time plots, particularly when comparing several methods. Furthermore, it can be difficult to discern if artificial viscosity or physical viscosity affects the frequency from these type of plots, especially in the cases where more than a single frequency is present. To elucidate some of these ideas, harmonic analysis was used to examine the detonation pressure-time series away from initialization. This type of analysis can reveal important information about and individual signal, such as at what frequency is the majority of the energy being transmitted. It is also powerful tool to analyze the differences and similarities between two signals.

To further clarify the issue of which frequencies are carrying energy of the detonation as well as as how this changes with overdrive, the power spectral density (PSD) was used. The PSD of a signal describes how the variance (or power) is distributed in frequency and it is real-valued for any real signal. It can be used to reveal possible periodicities in a complex signal, similar to what has been seen in the two mode case predicted at low overdrives. The variation between two signals can also be studied using PSD by making a comparison between the two spectra. The PSD is simply defined as the Fourier Transform of the autocorrelation of a signal. ${ }^{39,40}$ The autocorrelation function, $\phi(t)$, of the signal, $p(t)$, is defined as

$$
\phi(t)=\int_{-\infty}^{\infty} p(\tau+t) p(\tau) d \tau
$$

Therefore the PSD, $\Phi(\bar{f})$, is defined as

$$
\Phi(\bar{f})=\int_{-\infty}^{\infty} \phi(\tau) \exp (-2 \pi \imath \bar{f} \tau) d \tau
$$

where $\bar{f}$ is the frequency. Using the Wiener-Khinchin theorem, the Fourier Transform of the autocorrelation, Eqn. 23, can be written as the magnitude squared of the Fourier Transform of the signal. Thus the PSD is

$$
\Phi(\bar{f})=\left|\int_{-\infty}^{\infty} p(t) \exp (-2 \pi \imath \bar{f} t) d t\right|^{2}=|P(\bar{f})|^{2},
$$

where $P(\bar{f})$ is the Fourier Transform of $p(t)$.

The discrete, one-sided mean squared amplitude PSD was used in this study. In order to do this calculation, suppose we have $N$ equally spaced sample values of the detonation pressure-time signal, $p(t)$, such that

$$
p_{n}=p\left(t_{n}\right), \quad t_{n}=n \Delta t, \quad n=0,1,2, \ldots, N-1,
$$

then the standard discrete Fourier transform of $p$ at mode $k, P_{k}$, can be calculated by:

$$
P_{k}=\sum_{n=0}^{N-1} p_{n} \exp \left(-\frac{2 \pi \imath n k}{N}\right), \quad k=0,1,2, \ldots, N / 2
$$

where mode $k$ is associated with frequency, $\bar{f}_{k}$, which is defined only for the zero and positive frequencies as

$$
\bar{f}_{k}=\frac{k}{N \Delta t}, \quad k=0,1,2, \ldots, N / 2
$$

Then the discrete form of the one-sided, mean squared amplitude PSD, $\Phi_{d},{ }^{41}$ can be calculated as:

$$
\Phi_{d}(0)=\frac{1}{N^{2}}\left(P_{o} P_{o}\right)=\frac{1}{N^{2}}\left|P_{o}\right|^{2}
$$




$$
\begin{aligned}
& \Phi_{d}\left(\bar{f}_{k}\right)=\frac{1}{N^{2}}\left(P_{k} P_{k}+P_{N-k} P_{N-k}\right)=\frac{1}{N^{2}}\left(\left|P_{k}\right|^{2}+\left|P_{N-k}\right|^{2}\right) \approx \frac{2}{N^{2}}\left|P_{k}\right|^{2}, \quad k=1,2, \ldots,(N / 2-1), \\
& \Phi_{d}(N / 2)=\frac{1}{N^{2}}\left(P_{N / 2} P_{N / 2}\right)=\frac{1}{N^{2}}\left|P_{N / 2}\right|^{2} .
\end{aligned}
$$

The choice of the single-sided PSD was made in order to avoid the aliasing effect at high frequencies, otherwise Eqns. 28 are the discrete analog of Eqn. 24. The particular normalization chosen here, was done so that the sum of the $N / 2+1$ values of $\Phi_{d}$ is equal to the mean squared amplitude of the discrete pressure signal, $\frac{1}{N} \sum_{n=0}^{N-1}\left|p_{n}\right|^{2}$, by using Parseval's theorem.
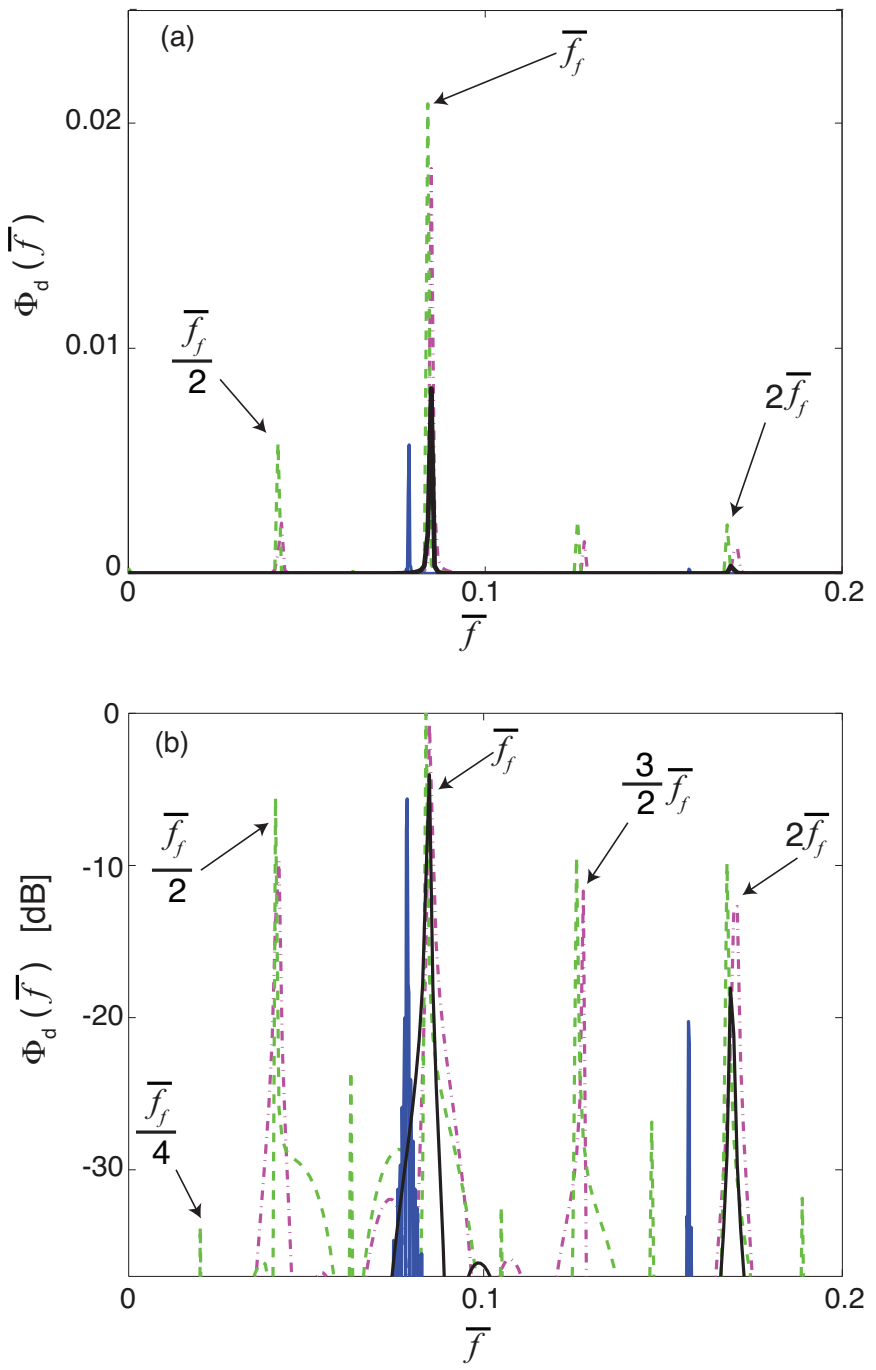

Figure 9: The long time behavior PSD for several activation energies using the one-step model. (a) Shows the non-dimensional PSD and (b) using decibels.

\section{One-step dynamics}

To ground the harmonic analysis in the context of a problem with well-known results from linear stability, let us examine a few results from one-step kinetics. From Lee and Stewart ${ }^{42}$ and Sharpe, ${ }^{43}$ as well as others, it is known that the first unstable mode for a CJ detonation in one-step kinetics, using ratio of specific heats of $\gamma=1.2$ and non-dimensional heat release of $Q=50$, occurs at an activation energy of $E_{a} \approx 25.265$. At any activation energy above this critical point the steady-state detonation profile is unstable in nature at long 
times. For this combination of heat release and a ratio of specific heats, only a single linearly unstable mode exists at activation energies below chaotic or nearly chaotic behavior. For example, at an activation energy of $E_{a}=26.00$, linear stability predicts a unstable mode at non-dimensional frequency of $\bar{f}_{f} \approx 0.0879 .{ }^{25}$ Henrick et $a l .{ }^{25}$ were able to match well at early times the linear stability frequency and growth rates. Furthermore at this activation energy in the inviscid limit, a period-1 detonation has been predicted at long times. ${ }^{25,44}$ Looking at the long time behavior at this activation energy through the frequency domain, it is clear from Fig. 9(a) that nearly all of the energy is contained at single dominant frequency at the defined spike in the PSD. The PSD presented is the non-dimensional power-frequency spectrum, where the steady ZND detonation pressure has been used to non-dimensional pressure. All results presented for the onestep kinetics were calculated using shock-fitting with 40 points in the half-reaction length. The predicted fundamental frequency at long times for this activation energy is $\bar{f}_{f}=0.0849$ which is a relative difference of $3.41 \%$ versus the linear stability frequency. The fundamental frequency is also known as the first harmonic of the system. This difference is attributed to the saturation of nonlinear effects at long times. From Fig. 9(b) it can be more easily ascertained that the harmonics of the fundamental mode also contain energy of the detonation, though showing a power law decrease in decibels. Here instead of the traditional definition of decibels $\left[10 \times \log _{10}(P S D / \max (P S D))\right]$, all spectra shown in Fig. 9(b) have been scaled by the maximum of the $E_{a}=27.7$ spectrum so the magnitude of the different spectra would not be lost. Both $\mathrm{Ng}$ et al. ${ }^{44}$

and Henrick et $a .^{25}$ report a sub-harmonic bifurcation process, where a lower frequency develops as the activation energy is increased. As an example, for an activation energy $E_{a}=27.5$, a pulsating detonation with two distinct peaks in the detonation pressure-time signal is predicted. The PSD demonstrates the appearance of this sub-harmonic frequency with some energy now being carried at the lower frequency. The predicted fundamental frequency of $\bar{f}_{f}=0.0842$, is $6.23 \%$ larger relative to that predicted by linear stability of 0.0793 . Similar results were reported by Henrick et al. ${ }^{25}$ for the period-1 and period-2 detonations, while using 80 points in the half-reaction length. The nonlinear effect on the frequency has increased slightly from the period-1 detonation at this higher activation energy, and is manifested in the multi-mode nature of the detonation. Using an eigenvalue decomposition of perturbations to a multi-dimensional detonation wave in the one-step model, Massa et $a l .{ }^{45}$ found that the least stable perturbations occurred near this first sub-harmonic suggesting that even in multiple dimensions the dominant mechanism is similar to that seen in galloping case of one dimension. Examining a just slightly higher activation energy of $E_{a}=27.7$, a period-4 detonation is formed indicated by the $\bar{f}_{f} / 4$ frequency present in Fig. 9(b). Examining the viscous analog of this particular activation energy, using the results from Romick et al., ${ }^{18}$ it can be seen that viscosity dramatically changes the predicted PSD, from a period-4 detonation to a period-1 behavior to accompany a shift in the fundamental frequency from 0.0839 in the inviscid case to 0.0787 , which is only $0.12 \%$ from the prediction of linear stability of 0.0786 . Moreover, there has also been a significant reduction in the amplitude of the peak in the spectrum.

\section{Hydrogen-air dynamics}

Similar to the behavior of the one-step kinetics model, as activation energy is increased, hydrogen-air detonations first form a high frequency instability, and then as the overdrive is lowered this is followed by the appearance of a lower frequency mode. However, unlike the one-step model the hydrogen-air detonations at these ambient conditions do not go through a period-doubling process so the lower frequency is not explicitly a sub-harmonic of the higher frequency. The PSDs presented are for the detonation pressure-time signal, where the pressure has been made non-dimensional using the steady state ZND pressure.

As stated in section $\mathrm{B}$, below a critical overdrive the detonation becomes unstable, and a high frequency pulsating detonation develops. For an overdrive $(f=1.12)$ just below this critical point, an oscillating detonation with a small amplitude of oscillation is predicted. The PSD in Fig. 10(a) is not able to capture this behavior, as the amplitude of oscillations at this overdrive is significantly smaller than the amplitudes for overdrives further below the neutral stability overdrive. However, looking at the the PSD in decibels, as in Fig. 10(b), a definitive spike can be seen at $1.03 \mathrm{MHz}$. Again it should be noted, that in order to not lose information about the magnitude of the spectrum the maximum of the $f=1.075$ spectrum was used for scaling. Even for a stable detonation $(f=1.15)$ there is a small peak; however, the magnitude of this peak is minute and would likely disappear completely examining even longer times. Examining overdrives further below the neutral stability point, the amplitude of oscillations are significantly larger than those near the stability point and continue to grow as the overdrive is lowered. Furthermore, as the overdrive is lowered the harmonics of the fundamental frequency also grow in amplitude as demonstrated in Fig. 10, 

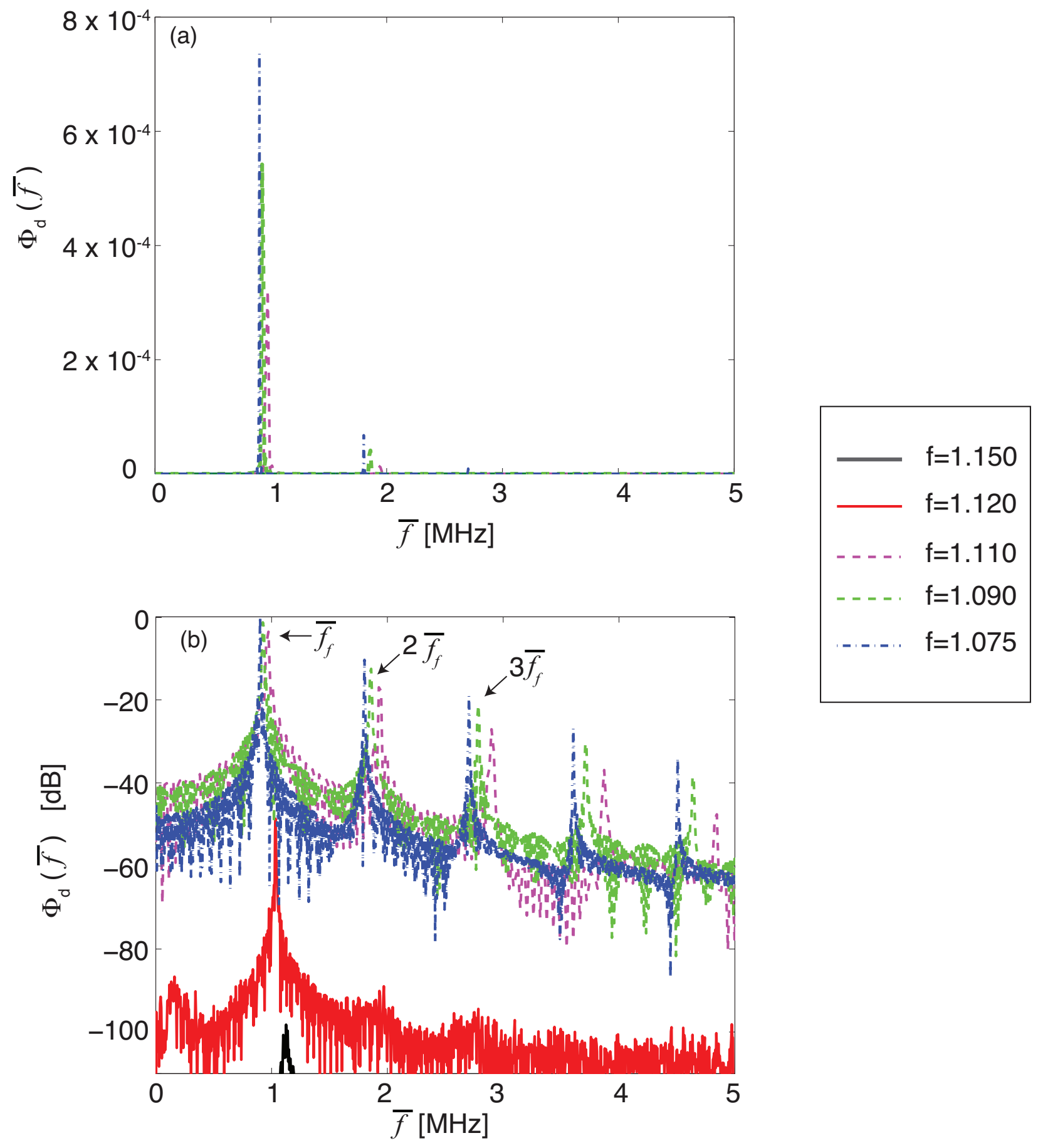

Figure 10: The long time behavior PSD for several overdriven hydrogen-air detonations with an ambient state of $0.421 \mathrm{~atm}$ and $293.15 \mathrm{~K}$ near the neutral stability point in the inviscid limit using shock-fitting with $\Delta x=4 \mu m$. (a) Shows the non-dimensional PSD and (b) shows the PSD in decibels. 
though the same power law decrease observed in the one-step model is also predicted in detailed kinetics. The concentration of the energy at a single frequency gives rise to the predicted regular oscillations seen at the fundamental frequency in this high frequency mode. As the overdrive is lowered, the location of the fundamental frequency shifts to lower frequencies. At an overdrive of $f=1.12$ the fundamental frequency predicted is $\bar{f}_{f}=1.03 \mathrm{MHz}$, whereas at $f=1.075$ the first harmonic has shifted to $\bar{f}_{f}=0.901 \mathrm{MHz}$.

The predicted higher harmonics, which for the high frequency mode are in the range of $1 \mathrm{MHz}$ to $5 \mathrm{MHz}$, are well below the cut-off frequency. In the inviscid case, the largest time-step used was on the order of $8 \times 10^{-10} \mathrm{~s}$. This time-step size yields a sampling frequency on the order of $1 \mathrm{GHz}$, and a cutoff frequency is near $500 \mathrm{M} \mathrm{Hz}$. At these much higher values of frequency, it maybe pertinent to examine the continuum limit. According with Vincenti and Kruger, ${ }^{24}$ the collision time $\tau_{c}=\lambda / \sqrt{\bar{C}^{2}}$, where $\bar{C}^{2}$ is the mean-square molecular speed, given by $\bar{C}^{2}=3 p / \rho$ and $\lambda$ the mean-free path. It was shown in section II that behind the typical detonation front studied here, that the mean-free path $\lambda \approx 3 \times 10^{-6} \mathrm{~cm}$. In the typical detonation studied, the front pressure is $\mathcal{O}(13 \mathrm{~atm})$, and front density $\mathcal{O}\left(2 \times 10^{-3} \mathrm{~g} / \mathrm{cm}^{3}\right)$. Therefore, the collision times at the detonation front are $\mathcal{O}\left(10^{-11} \mathrm{~s}\right)$; however, for the continuum model to hold, several of these collision times must pass. Thus in the continuum limit, any predicted frequency above approximately $10 \mathrm{GHz}$ must be discarded as non-physical; this limit is well above the predicted fundamental frequency and its harmonics. Furthermore, a more stringent time-scale to consider is the vibrational relaxation times at the detonation front as these can be far larger than the collision times. A correlation, found by Dove and Teitelbaum, ${ }^{46}$ gives the vibrational relaxation times of the $H_{2}-H_{2}$ interaction on the order of $10^{-7} \mathrm{~s}$, for the typical detonation front. Therefore, any predicted frequency above $10 \mathrm{M} \mathrm{Hz}$ would likely be amiss in neglecting the vibrational relaxation explicitly. As seen in Fig. 10 (b), there is a power law decrease in the amount of energy the higher harmonics carry. The frequencies near the relaxation time limit are around the tenth harmonic and are predicted to carry a small amount of energy.

One can calculate a rough estimate of the length scales for these oscillations in a manner similar to that given by Al-Khateeb et al. ${ }^{20}$ who estimate the length scale as $\ell=\sqrt{D_{i k}}$. For example, the fine diffusion scale diffusion mode is estimated by using the smallest diffusion coefficient, $D_{i k} \approx \mathcal{O}\left(10^{1} \mathrm{~cm}^{2} / \mathrm{s}\right)$, and higher harmonics' time scale and thus yields $\ell \approx \mathcal{O}\left(10^{-4} \mathrm{~cm}\right)$. Likewise using largest diffusion coefficient, $D_{i k} \approx \mathcal{O}\left(10^{2} \mathrm{~cm}^{2} / \mathrm{s}\right)$, and the first harmonic's time scale, a coarsest diffusion length scale can be calculated, and gives a coarsest length scale estimate is $\ell \approx \mathcal{O}\left(10^{-2} \mathrm{~cm}\right)$. This diffusion length scale is similar to that predicted when using advection speeds in the detonation products, $u=\mathcal{O}\left(1 \times 10^{5} \mathrm{~cm} / \mathrm{s}\right)$, and the time scales from the harmonic analysis. The higher harmonics near $5 M H z$ yield a wavelength of $\ell=u \tau \approx \mathcal{O}\left(10^{-2} \mathrm{~cm}\right)$. The harmonics above this frequency, as stated earlier, have small amplitude peaks and thus carry only a small amount of the overall energy.

As the second critical overdrive is approached, the pulsations begin to exhibit multiple frequencies. Below this critical point, the long time behavior becomes dominated by the low frequency $(\sim 0.1 \mathrm{MHz})$ mode and the majority of the energy is carried in lower frequencies, as seen in Fig. 11(a). Examining the long times $(t>150 \mu s)$ of several lower overdrives in the frequency domain, it becomes clear that the amplitude growth of the oscillations is accompanied by a significant shift in the fundamental frequency. At the neutral stability point, the fundamental frequency is near $1 \mathrm{MHz}$ and it continuously shifts to lower frequencies as the overdrive is lowered. Eventually reaching a frequency of $0.713 \mathrm{MHz}$ at $f=1.035$. Below this point, there is a drastic change in the fundamental frequency to $0.110 \mathrm{MHz}$ at $f=1.029$, as shown by Fig. 11(b). However, the second largest peak in the PSD does not occur at the second harmonic of the fundamental frequency during this transition region, but rather it stays near $0.591 \mathrm{MHz}$. This indicates that there is a clear competition between the two modes. The second harmonic becomes the second largest energy content holder at an overdrive of $f=1.018$ which has a fundamental frequency of $\bar{f}_{f}=0.105 \mathrm{MHz}$. Even at this low overdrive, the detonation exhibits the high frequency mode at early times as indicated by the temporal evolution shown in Fig. 12(a). However at this overdrive the transition from the high to low frequency happens quickly, as indicated by the PSD shown in Fig. 12(b). At early time, the PSDs show a large spike near $0.7 \mathrm{MHz}$; however, as seen in the temporal evolution, the strength of the high frequency mode weakens. In the second PSD, for the time range of $50<t<150 \mu \mathrm{s}$, the low frequency is already dominant. Furthermore at long time, the PSD shows very strong peaks at $0.105 \mathrm{MHz}$ and at the second harmonic indicating most of the energy is being carried at these two frequencies.

EFFECT OF SHOCK-CAPTURING The results of the more common technique of shock-capturing technique were compared with those from the negligible artificial viscosity shock-fitting technique. For an overdrive of $f=1.10$, the long time PSDs were computed for shock-capturing using $\Delta x=1 \mu \mathrm{m}$ and $\Delta x=1 / 2 \mu \mathrm{m}$ 

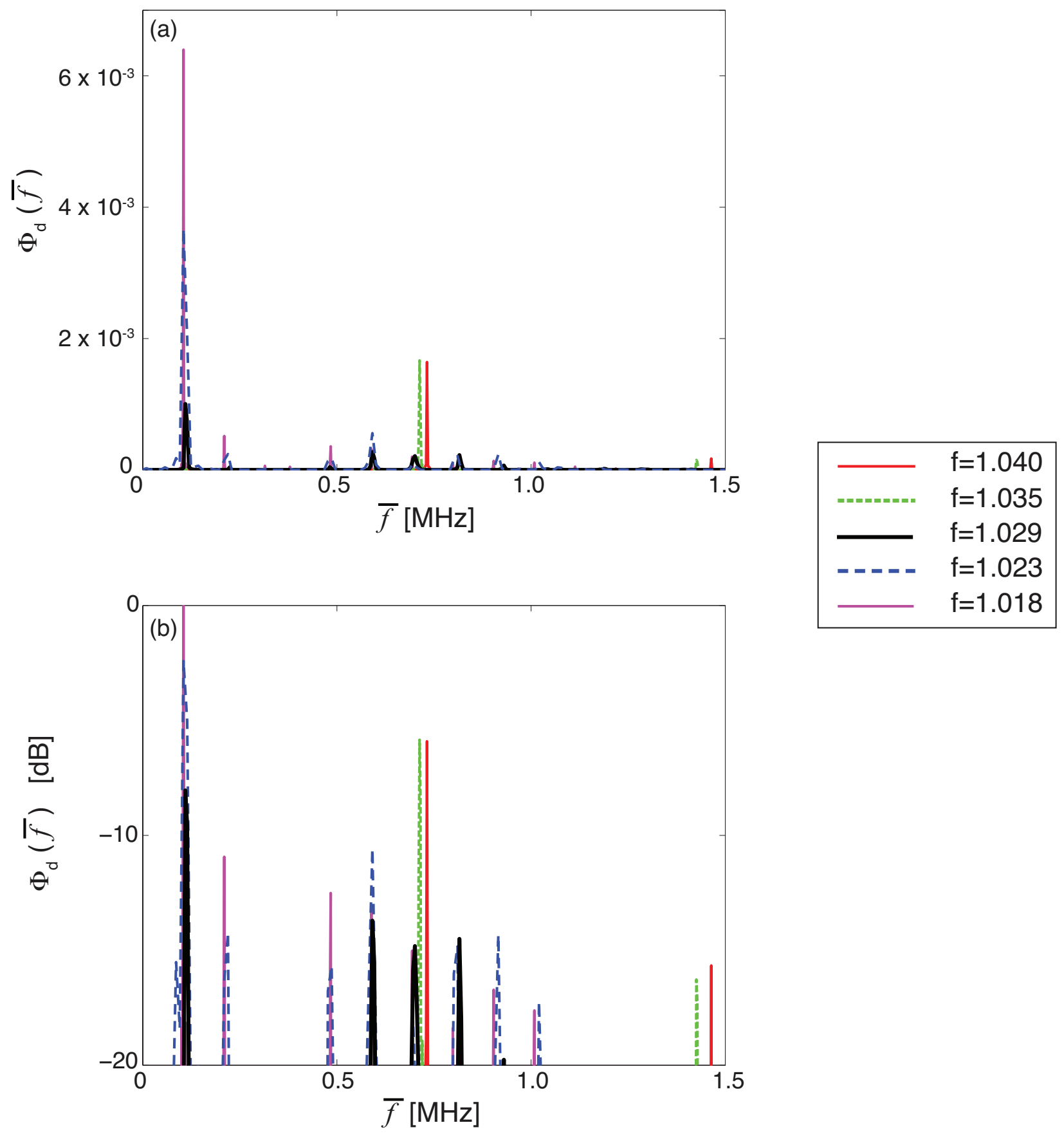

Figure 11: The long time behavior PSD for several overdriven hydrogen-air detonations with an ambient state of $0.421 \mathrm{~atm}$ and $293.15 \mathrm{~K}$ near the second bifurcation point in the inviscid limit using shock-fitting with $\Delta x=4 \mu \mathrm{m}$. (a) Shows the non-dimensional PSD and (b) shows the PSD in decibels. 

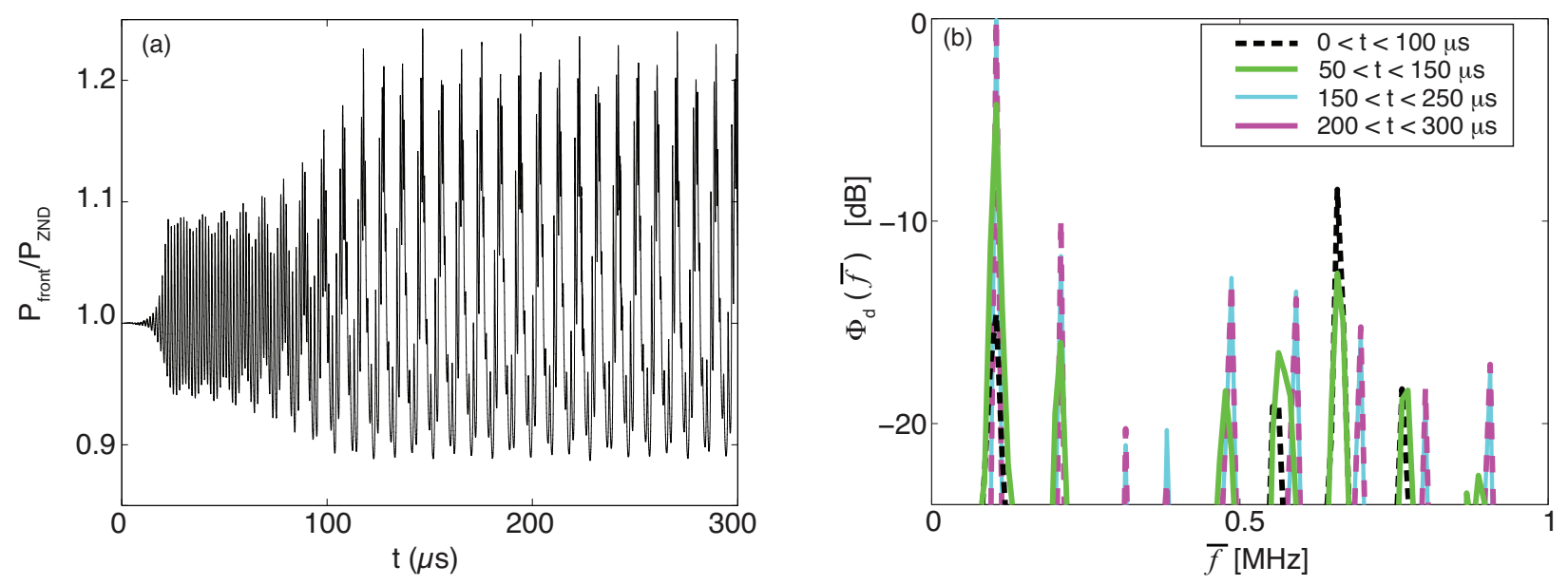

Figure 12: The behavior for an overdriven, $f=1.018$, hydrogen-air detonation with an ambient state of $0.421 \mathrm{~atm}$ and $293.15 \mathrm{~K}$ in the inviscid limit using shock-fitting and $\Delta x=4 \mu \mathrm{m}$ in the (a) time-domain and (b) frequency domain for several ranges in time.

in a moving reference frame, $\Delta x=1 \mu m$ in a non-moving frame of reference as well as using $\Delta x=4 \mu m$ and $\Delta x=2 \mu m$ with shock-fitting, and Fig. 13(a) shows zoomed profile near the predicted fundamental frequency. As seen earlier, at the coarser grids the shock-capturing technique smears the detonation front enough to cause the predicted behavior to be stable at long times and thus those PSDs are not shown here. By zooming in on the first harmonic, more detailed information can be gathered about the different predictions. As indicated in section $\mathrm{B}$, there is modification of the predicted fundamental frequency using the shock-capturing in a non-moving frame, but only by $1 \%$. However, the amplitude matches well with both the shock-fitting results and the finest resolution used in shock-capturing in a moving reference frame. Shockcapturing utilizing a micron grid size in a moving reference frame under predicts the amplitude of oscillation slightly, but predicts the fundamental frequency well. Since shock-fitting enforces the jump conditions, it can properly capture the dynamics of the detonation at coarser grids. It gives a prediction of $\bar{f}_{f}=0.928 \mathrm{MHz}$ at the four micron resolution and $\bar{f}_{f}=0.925 \mathrm{MHz}$ at the two micron resolution in agreement with shockcapturing at a half micron resolution in a moving reference frame, which predicts $\bar{f}_{f}=0.925 \mathrm{MHz}$.

When the dynamics are more complex at lower overdrives, the comparison between shock-fitting and shock-capturing becomes more difficult in the time domain as demonstrated by Fig. 8. The long time PSD for several cases of shock-capturing are compared to shock-fitting at an overdrive of $f=1.023$, shown in Fig. 13(b). At this overdrive, the coarsest grids used in the shock-capturing scheme dramatically over-predict the amplitude of pulsations, as well as mis-predicting the pulsation frequency. At a micron grid resolution, as in the higher overdrive case, the shock-capturing results begin to correspond to those predicted using shockfitting with a grid four times as coarse. The PSD is rather coarse for all cases considered here indicating a longer time sample should be taken in the future to further validate these findings.

EFFECT OF PHYSICAL DIFFUSION Several overdrives were examined to illustrate the long time effects of adding physical viscosity to the model of a detonation with detailed kinetics. The PSDs were calculated in decibels (using the respective inviscid maximum to calculate the diffusive spectrum) for overdrives ranging from $1.035 \leq f \leq 1.12$ are shown in Fig. 14. The frequency predicted by the inviscid calculations largely remains unchanged by adding physical diffusion when the viscous analog shows the same mode, even at the most unstable condition examined $(f=1.035)$ where multiple frequencies persist at longer times, shown in Fig. 14(d). The largest modulation in the fundamental frequency predicted was $1.3 \%$ at $f=1.055$, which is shown in Fig. 14(c). However near the stability boundary $(f=1.12)$, the predicted behavior frequency is significantly altered by the addition of viscosity. In fact the small pulsations are damped completely at long time, thus the diffusive PSD shows no resemblance to that of inviscid case in Fig. 14(a). In all cases examined the magnitude of the fundamental frequency in the PSD is decreased by the addition of physical viscosity in all cases tested. Furthermore, not only does diffusion reduce the first harmonic's magnitude, but it also reduces the magnitude of the second harmonic. In fact, the second harmonic's magnitude is 

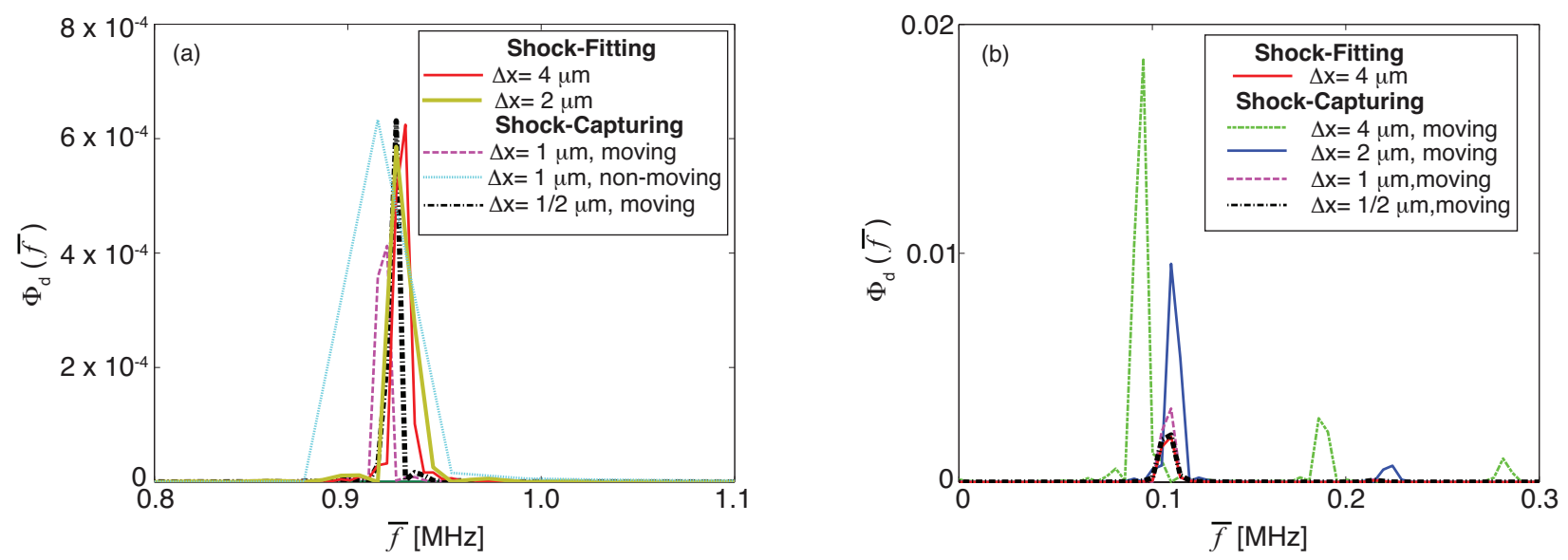

Figure 13: The PSD for an overdriven, hydrogen-air detonation with an ambient state of 0.421 atm and $293.15 K$ in the inviscid limit using both shock-capturing and shock-fitting showing the fundamental frequency for (a) $f=1.10$ and (b) $f=1.023$.

reduced more than the that of the first harmonic, indicating diffusion is making the detonations more regular. This reduction effect on the magnitude of the peaks decreases as the overdrive is lowered. The lowest overdrive examined shows the strongest correlation between the viscous and inviscid predictions, as shown in Fig. 14(d). This indicates that as the intrinsic instability of the detonation, which caused by the interaction of the hydrodynamics and chemical reactions, grows in strength the addition of physical viscosity has smaller effect, though longer times need to be further examined as indicated in section C.

\section{Conclusions}

An investigation of one-dimensional, unsteady, overdriven, hydrogen-air detonations has shown that as the initial overdrive is decreased, the long time behavior of the detonation becomes more complex, which is consistent with previous studies. ${ }^{4,7,8}$ Three distinct phases in the long time behavior were found for an initially stoichiometric hydrogen-air mixture at $0.421 \mathrm{~atm}$ and $293.15 \mathrm{~K}$. At high overdrives, the detonation is stable. Below a critical overdrive, a pulsating detonation is achieved with a single frequency predicted. As the overdrive is lowered the frequency at which the detonation pulsates shifts to lower frequencies and the amplitude of the pulsations grow. Lowering the overdrive yet further, a second critical overdrive is found below which the pulsating detonation begins to show multiple frequencies and much larger pulsations. In addition, the predicted $0.97 \mathrm{MHz}$ frequency for a $f=1.10$ overdriven detonation agrees with the frequency of $1.04 \mathrm{MHz}$ observed by Lehr in his experiments of shock-induced combustion flow around spherical projectiles in a hydrogen-air mixture at a inflow corresponding to a similar overdrive.

Harmonic analysis was used to examine the frequency domain and energy content of the detonation cycles. Furthermore, the PSD for the long-time behavior was calculated at various overdrives to examine how the long-time energy variance changes with the initial overdrive of the detonation. As the overdrive is lowered, the magnitude of the main peak (first harmonic) grows in strength indicating that the strength of the instability is growing as overdrive is lowered. Additionally, the location of the first harmonic shifts to lower frequencies signifying the period of the repeating detonation is expanding as the overdrive is lowered. At the second bifurcation in behavior, there is drastic change in the fundamental frequency and a second frequency below that predicted for higher overdrives appears. This change occurs at an overdrive of $f=1.029$, with the frequency changing from $0.71 \mathrm{MHz}$ to $0.11 \mathrm{MHz}$. This is similar to what is seen in the one-step model as the activation energy is increased. However, unlike the one-step kinetics model the hydrogen-air does not undergo a period doubling process at these ambient conditions. Moreover, this lower frequency is not an explicit sub-harmonic of the fundamental frequency. It was also found that a transition region exists in which the two main frequencies persist at long times.

The common use of shock-capturing was examined and compared with shock-fitting, and it was found that in the unstable regime a finer resolution was necessary to capture the dynamics of the detonation. 

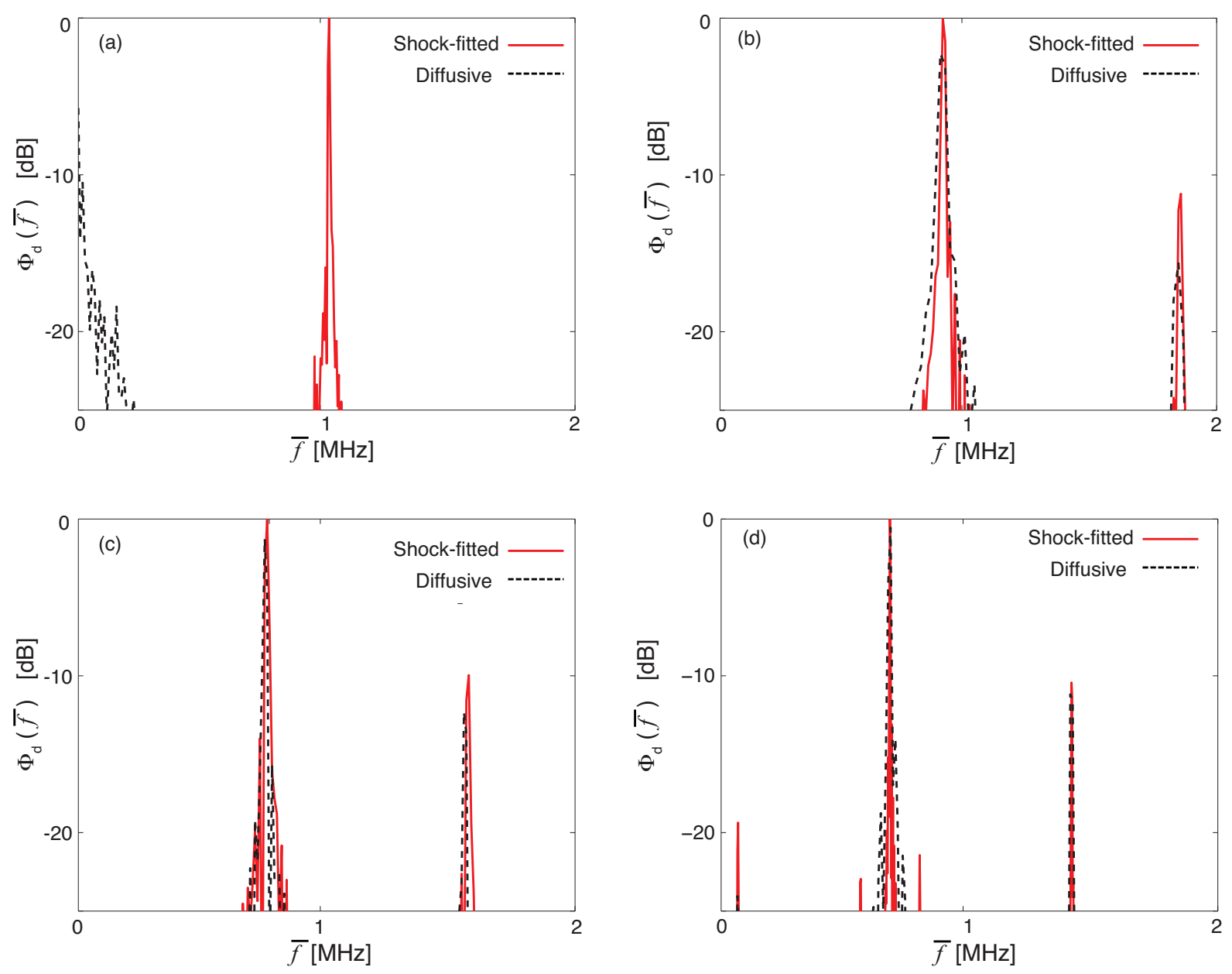

Figure 14: The PSD for both the inviscid and viscous predictions at long times for hydrogen-air detonation at overdrives of (a) $f=1.12$, (b) $f=1.09$, (c) $f=1.055$, and (d) $f=1.035$.

In both cases presented here, a four times finer grid was necessary to predict the essential dynamics with shock-capturing than shock-fitting. It was also demonstrated that using shock-capturing in moving reference frame gives rise to spurious oscillations at coarser grids and care needs to be taken to not give a physical interpretation of these non-physical oscillations.

The inclusion of physical mass, momentum and energy has been seen to have a stabilizing effect on the long time behavior in all unstable cases examined. The PSD comparison of the inviscid and viscous predictions shows an overall reduction in the peak magnitude at all cases examined, including at overdrives leading mixed mode detonations. Furthermore, the amount of energy contained at the higher harmonics is reduced in a similar way to that of the first harmonic frequency. The magnitude reduction effect is weakened as the overdrive is lowered closer to the CJ detonation velocity for the cases examined here. This is caused by the increase in the strength of the intrinsic instability, due to the interaction of the hydrodynamics and the chemical reactions. Away from the bifurcation limits, it appears that the addition of physical viscosity has a minimal effect on the predicted fundamental frequency. Yet, further investigation is needed near the bifurcation limits, to illustrate how these points are altered by the addition of physical viscosity due to nonlinear effects. Additionally, further cases will be examined for the longer time behavior and lower overdrives to gain further insight into the effect of physical diffusion and its importance near the second bifurcation in behavior. 


\title{
Acknowledgments
}

This work was performed under support of the US Department of Energy.

\section{References}

\begin{abstract}
${ }^{1}$ Powers, J. M., and Paolucci, S., "Accurate Spatial Resolution Estimates for Reactive Supersonic Flow with Detailed Chemistry," AIAA Journal, Vol. 43, No. 5, 2005, pp. 1088-1099.

${ }^{2}$ Al-Khateeb, A. N., Powers, J. M., and Paolucci, S., "On the Necessary Grid Resolution for Verified Calculation of Premixed Laminar Flames," Communications in Computational Physics, Vol. 8, No. 2, 2010, pp. 304-326.

${ }^{3}$ Shepherd, J. E., "Detonation in gases," Proceedings of the Combustion Institute, Vol. 32, 2009, pp. 83-98.

${ }^{4}$ Sussman, M .A., "Numerical Simulation of Shock-Induced Combustion," Ph. D. Dissertation, Department of Aeronautics and Astronautics, Stanford University, Stanford, CA, 1995.

${ }^{5}$ Eckett, C. A., "Numerical and Analytical Studies of the Dynamics of Gaseous Detonations," Ph. D. Dissertation, California Institute of Technology, Pasadena, CA, 2001.

${ }^{6}$ Singh, S., Rastigejev, Y., Paolucci, S., and Powers, J. M., "Viscous detonation in $\mathrm{H}_{2}-\mathrm{O}_{2}-\mathrm{Ar}$ Using Intrinsic LowDimensional Manifolds and Wavelet Adaptive Multilevel Representation," Combustion Theory and Modelling, Vol. 5, No. 2,
\end{abstract} 2001, pp. 163-184.

${ }^{7}$ Yungster, S., and Radhakrishan, K., "Pulsating one-dimensional detonations in hydrogen-air mixtures," Combustion Theory and Modelling, Vol. 8, No. 4, 2004, pp. 745-770.

${ }^{8}$ Daimon, Y., and Matsuo, A., "Unsteady Features on One-Dimensional Hydrogen-Air Detonations," Physics of Fluids, Vol. 19, No. 11, 2007, Art. No. 116101.

${ }^{9}$ Oran, E. S., Weber, J. W., Stefaniw, E. I., Lefebvre, M. H., and Anderson, J. D., "A Numerical Study of a TwoDimensional $\mathrm{H}_{2}-\mathrm{O}_{2}-\mathrm{Ar}$ Detonation Using a Detailed Chemical Reaction Model," Combustion and Flame, Vol. 113, No. 1-2, 1998, pp. 147-163.

${ }^{10} \mathrm{Hu}$, X. Y., Khoo, B. C., Zhang, D. L., and Jiang, Z. L., "The Cellular Structure of a Two-Dimensional $H_{2} / O_{2} / A r$ Detonation Wave," Combustion Theory and Modelling, Vol. 8, No. 2, 2004, pp. 339-359.

${ }^{11} \mathrm{Hu}$, X. Y., Zhang, D. L., Khoo, B. C., and Jiang, Z. L., "The Structure and Evolution of a Two-Dimensional $H_{2} / O_{2} / A r$ Cellular Detonation," Shock Waves, Vol. 14, No. 1-2, 2005, pp. 37-44.

${ }^{12}$ Ziegler, J. L., Deiterding, R., Shepherd, J. E., and Pullin, D. I., "An Adaptive High-Order Hybrid Scheme for Compressive, Viscous Flows with Detailed Chemistry," Journal of Computational Physics, Vol. 230, No. 20, 2011, pp. 7598-7630.

${ }^{13}$ Taylor, B. D., Kessler, D. A., Gamezo, V. N., and Oran, E. S., "The Influence of Chemical Kinetics on the Structure of Hydrogen-Air Detonations," 50th AIAA Aerospace Sciences Meeting and Exhibit, AIAA, 2012, Nashville, TN, paper AIAA2012-0979.

${ }^{14}$ Tsuboi, N., Eto, K., and Hayashi, A. K., "Detailed Structure of Spinning Detonation in a Circular Tube," Combustion and Flame, Vol. 149, No. 1-2, 2007, pp. 144-161.

${ }^{15}$ Deiterding, R., "A Parallel Adaptive Method for Simulating Shock-Induced Combustion with Detailed Chemical Kinetics in Complex Domains," Computers and Structures, Vol. 87, No. 11-12, 2009, pp. 769-783.

${ }^{16}$ Powers, J. M., "Review of Multiscale Modeling of Detonation", Journal of Propulsion and Power, Vol. 22, No. 6, 2006, pp. $1217-1229$.

${ }^{17}$ Kivotides, D., "Viscous Microdetonation Physics," Physics Letters A, Vol. 363, No. 5-6, 2007, pp. $458-467$.

${ }^{18}$ Romick, C. M., Aslam, T. D., and Powers, J. M., "The Effect of Diffusion on the Dynamics of Unsteady Detonations," Journal of Fluid Mechanics, Vol. 699, 2012, pp. 453-464.

${ }^{19}$ Mazaheri, K., Mahmoudi, Y., and Radulescu, M. I., "Diffusion and Hydrodynamic Instabilities in Gaseous Detonations," Combustion and Flame, Vol. 159, No. 6, 2012, pp. 2138-2154.

${ }^{20}$ Al-Khateeb, A. N., Powers, J. M., and Paolucci, S., "Analysis of the Spatio-Temporal Scales of Laminar Premixed Flames Near Equilibrium," Combustion Theory and Modelling, To Appear, doi = 10.1080/13647830.2012.724180, URL = http://www.tandfonline.com/doi/abs/10.1080/13647830.2012.724180.

${ }^{21}$ Merk, H. J., "The Macroscopic Equations for Simultaneous Heat and Mass transfer in Isotropic, Continuous and Closed systems," Applied Scientific Research. Vol. 8, No. 1, 1959, pp. 73-99.

${ }^{22}$ Kee, R. J., Dixon-Lewis, G., Warnatz, J., Coltrin, M. E., and Miller, J. A., "A Fortran Computer Code Package for the Evaluation of Gas-Phase Multi-Component Transport Properties," Sandia National Laboratories, SAND86-8246, Albuquerque, NM, 1991.

${ }^{23}$ Kee, R. J., Rupley, F. M., and Miller, J. A., "Chemkin II: A Fortran Chemical Kinetics Package for the Analysis of Gas Phase Chemical Kinetics," Sandia National Laboratories, SAND89-8009B, Albuquerque, NM, 1992.

${ }^{24}$ Vincenti, W. G., and Kruger, C. H., Introduction to Physical Gas Dynamics, Wiley, 1967.

${ }^{25}$ Henrick, A. K., Aslam, T. D., and Powers, J. M., "Simulations of Pulsating One-Dimensional Detonations with True Fifth Order Accuracy," Journal of Computational Physics, Vol. 213, No. 1, 2006, pp. 311-329.

${ }^{26}$ Vasilyev, O. V., and Paolucci, S., "Dynamically Adaptive Multilevel Wavelet Collocation Method for Solving Partial Differential Equations in a Finite Domain," Journal of Computational Physics, Vol. 125, No. 2, 1996, pp. 498-512.

${ }^{27}$ Vasilyev, O. V., and Paolucci, S., "A Fast Adaptive Wavelet Collocation Algorithm for Multidimensional PDEs," Journal of Computational Physics, Vol. 138, No. 1, 1997, pp. 16-56.

${ }^{28}$ Rastigejev, Y. A., "Multiscale Computations with a Wavelet Adaptive Algorithm," Ph. D. Dissertation, Department of Aerospace and Mechanical Engineering, University of Notre Dame, Notre Dame, IN, 2002. 
${ }^{29}$ Rastigejev, Y. A., and Paolucci, S., "Wavelet-Based Adaptive Multiresolution Computation of Viscous Reactive Flows," International Journal for Numerical Methods in Fluids, Vol. 52, No. 7, 2006, pp. 749-784.

${ }^{30}$ Rastigejev, Y. A., and Paolucci, S., "Wavelet Adaptive Multiresolution Representation: Applications to Viscous Multiscale Flow Simulations," International Journal of Wavelets, Multiresolution, and Information Processing, Vol. 4, No. 2, 2006, pp. 333-343.

${ }^{31}$ Wirasaet, D., and Paolucci, S., "An Adaptive Wavelet Method for Incompressible Flows in Complex Domains," Journal of Fluids Engineering, Vol. 127, No. 4, 2005, pp. 656-665.

${ }^{32}$ Wirasaet, D., "Numerical Solutions of Multidimensional Partial Differential Equations Using an Adaptive Wavelet Method," Ph. D. Dissertation, Department of Aerospace and Mechanical Engineering, University of Notre Dame, Notre Dame, IN, 2008.

${ }^{33}$ Zikoski, Z. J., "A Parallel Adaptive Wavelet Method for Multidimensional Simulations of Hypersonic Propulsion," Ph. D. Dissertation, Department of Aerospace and Mechanical Engineering, University of Notre Dame, Notre Dame, IN, 2011.

${ }^{34}$ Paolucci, S., Zikoski, Z., and Wirasaet, D., "WAMR: An Adaptive Wavelet Method for the Simulation of Compressible Reactive Flow. Part I. Accuracy and Efficiency of the Algorithm," Journal of Computational Physics, submitted, 2012.

${ }^{35}$ Paolucci, S., Zikoski, Z., and Grenga, T., "WAMR: An Adaptive Wavelet Method for the Simulation of Compressible Reactive Flow. Part II. The Parallel Algorithm," Journal of Computational Physics, submitted, 2012.

${ }^{36}$ Lehr, H. F., "Experiments on Shock-Induced Combustion," Astronautica Acta, Vol. 17, No. 4-5, 1972, pp. 589-597.

${ }^{37}$ Miller, J. A., Mitchell, R. E., Smooke, M. D., and Kee, R. J., "Toward a Comprehensive Chemical Kinetic Mechanism for the Oxidation of Acetylene: Comparison of Model Predictions with Results from Flame and Shock Tube Experiments," Nineteenth Symposium (International) on Combustion, The Combustion Institute, 1982, Haifa, Israel, pp. 181-196.

${ }^{38}$ Quirk, J. J., "A Contribution to the Great Riemann Solver Debate," International Journal for Numerical Methods in Fluids, Vol. 18, No. 6, 1994, pp. 555-574.

${ }^{39}$ Hamilton, J. D., Time Series Analysis, Princeton University Press, Princeton, NJ, 1994.

${ }^{40}$ Brillinger, D. R., Time Series: Data Analysis and Theory, Society for Industrial and Applied Mathematics, Philadelphia, PA, 2001.

${ }^{41}$ Oppenheim, A. V., and Schafer, R. W., Digital Signal Proccessing, Prentice-Hall, Englewood Cliffs, NJ, 1975.

${ }^{42}$ Lee, H. I., and Stewart, D. S., "Calculation of Linear Detonation Instability: One-dimensional Instability of Planar Detonations," Journal of Fluid Mechanics, Vol. 216, 1990, pp. 103-132.

${ }^{43}$ Sharpe, G. J., "Linear Stability of Idealized Detonations," Proceedings of the Royal Society of London Series A Mathematical Physical and Engineering Sciences, Vol. 453, No. 1967, 1997, pp. 2603-2625.

${ }^{44} \mathrm{Ng}$, H. D., Higgins, A. J.,Kiyanda, C. B., Radulescu, M. I., Lee, J. H. S., Bates, K. R., and Nikiforakis, N., "Nonlinear Dynamics and Chaos Analysis of One-Dimensional Pulsating Detonations," Combustion Theory and Modeling, Vol. 9, No. 1, 2005, pp. 159-170.

${ }^{45}$ Massa, L., Kumar, R., and Ravindran, P., "Dynamic Mode Decomposition Analysis of Detonation Waves," Physics of Fluids, Vol. 24, No. 6, 2012, Art. No. 066101.

${ }^{46}$ Dove, J. E., and Teitelbaum, H., "The Vibrational Relaxation of $H_{2}$. I. Experimental Measurements of the Rate of Relaxation by $\mathrm{H}_{2}, \mathrm{He}, \mathrm{Ne}, \mathrm{Ar}, \mathrm{Kr}, "$ Chemical Physics, Vol. 6, No. 3, 1974, pp. 431-444. 